\title{
Macro- and micro-modeling of crack propagation in encapsulation-based self-healing materials: Application of XFEM and cohesive surface techniques
}

\author{
F.A. Gilabert ${ }^{\mathrm{a}, \mathrm{b}, *}$, D. Garoz ${ }^{\mathrm{a}, \mathrm{b}}$, W. Van Paepegem ${ }^{\mathrm{a}}$ \\ a Ghent University, Department of Materials, Textiles and Chemical Engineering, Tech Lane Ghent Science Park - Campus A, Technologiepark-Zwijnaarde 903, Zwijnaarde \\ (Ghent) 9052, Belgium \\ b SIM vzw, Technologiepark 935, Zwijnaarde 9052, Belgium
}

\section{A R T I C L E I N F O}

\section{Keywords:}

Self-healing materials

Micro-capsules

Crack propagation

Debonding

Cohesive zone

Extended finite element method

\begin{abstract}
A B S T R A C T
Encapsulation-based materials are produced introducing some small healing fluid-filled capsules in a matrix. These materials can self-heal when internal cracks intercept and break the capsules. If the healing agent is released, the crack can be sealed. However, this is not always the case. These capsules need to be designed with the adequate shape and material to be properly broken. This paper presents two application models based on the combination of eXtended Finite Element Method (XFEM) elements and Cohesive Surfaces technique (CS) to predict crack propagation. Two types of encapsulated systems are considered: a concrete beam in a three-point bending test, and a micro-scale model of a representative volume element of a polymer subjected to a uniaxial tensile test. Despite both systems relying on different capsule shapes and different constituent materials, the models predict a similar non-linear response of the overall material strength governed by the coupled effect of the interface strength and the capsule radii-to-thickness ratio. Furthermore, even if an inadequate material and geometry combination is used, it is found that the mere presence of capsules might achieve, under certain conditions, an interesting overall reinforcement effect. This effect is discussed in terms of clustering and volume fraction of capsules.
\end{abstract}

G R A P H I C A L A B S T R A C T
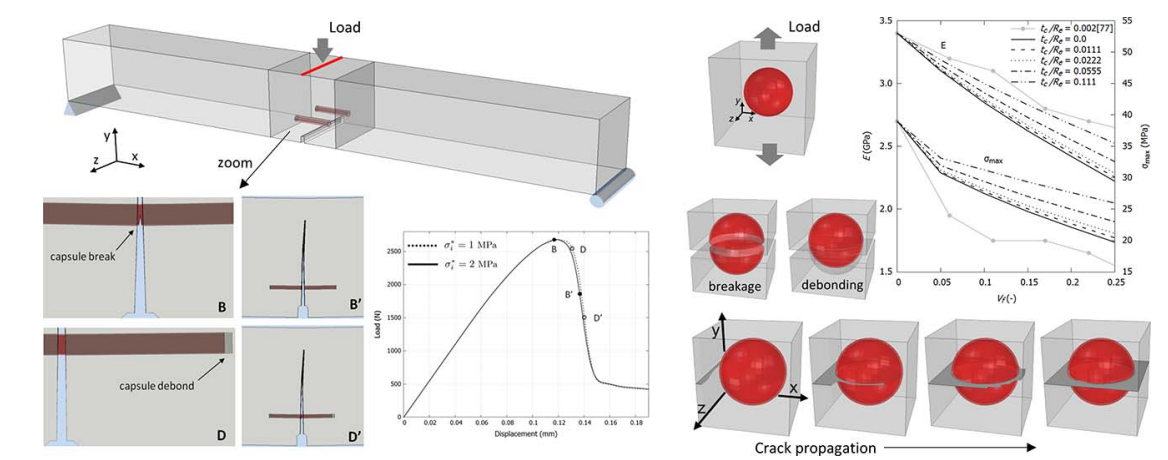

\section{Introduction}

The fundamental feature of any encapsulation-based self-healing material relies on inserting small fluid-filled capsules in a matrix [1-5].
When the matrix is internally damaged, cracks can intercept these capsules and transfer the stresses at the their propagating fronts to the capsules. In that sense, three basic scenarios can be produced: (i) the capsules are not able to withstand the stress concentration and they

\footnotetext{
" Corresponding author.

E-mail address: fran.gilabert@ugent.be (F.A. Gilabert).
} 
break, releasing in this way the healing fluid content inside the crack space, (ii) the constitution of the capsules is robust enough as to withstand comfortably much higher stresses, while the interface with the the matrix fails, and (iii) the level of transferred stresses is not sufficient either to break the capsule nor the interface with the matrix. Naturally, (i) represents the desired scenario for this self-healing strategy. In this case, the healing agent can spread into the crack space via capillarity. To complete the healing process, this initially-fluid agent can cure after some reaction, in such a way that the crack path becomes sealed and the final internal structure is partially repaired [6]. If the scenarios (ii) or (iii) occur, it is clear that this self-healing strategy cannot be carried out successfully. In particular, (ii) involves that an incoming crack will simply trigger a premature debonding, similarly to what happens when a solid inclusion is weakly bonded to a matrix $[7,8]$. Also, even if premature capsule debonding takes place before the crack interception, a hole-liked region could be created and therefore additional nearby cracks would be attracted. These cracks would simply pass along the capsule perimeter and continue their path again through the matrix $[9,10]$. This situation would not only result in an intact capsule preventing the internal repairing, but it might also accelerate the level of damage in the matrix. Regarding scenario (iii), albeit no healing effect would be triggered a priori, it might still produce some beneficial aspects in terms of mechanical reinforcement. Nevertheless, this reinforcement effect would naturally depend on the characteristics and properties of each constituent, as well as on the mechanical performance of the interface.

In terms of designing a predictive tool, the interest in encapsulationbased self-healing strategy is currently leading to an increase of theoretical and numerical works with the aim of getting a better understanding of its mechanisms and determining the key factors to improve efficiency and feasibility [11-15]. In spite of this, most models deal with very specific application-oriented materials and also assume specific geometries, boundary conditions and loading configuration. Moreover, the mere presence of the capsule-matrix interface has received less attention, when not neglected. As an example, within the context of cementitious materials, even when the interface has been accounted for, these models often assume a certain range of typical values of the interface properties, which are selected as a function of mechanical similarities given by composition or the degree of brittleness $[16,17]$.

The motivation of this work is to propose a combination of flexible and efficient enough numerical methodologies to assess, predict and investigate different mechanical aspects of an encapsulation-based structure. Simulating such structure, the researcher can analyze different combinations of materials and levels of load that might be probably difficult to implement and/or measure experimentally. Therefore, the prediction of the aforementioned scenarios could be analyzed, as well as any other situation that might not be expected in advance. This methodology is based on several well-established computational approaches in the context of fracture mechanics, the extended finite element method (XFEM) and the surface-based cohesive technique (CS), and both are combined to the conventional finite element method to describe regions where fracture mechanics is not needed. The mechanical properties required by these approaches, as well as the way to measure them can be experimentally identified with an acceptable level of complexity.

There are some recent numerical studies combining XFEM and cohesive zone models. Ref. [18] presents a similar approach where a micro-scaled piece of a unidirectional carbon-reinforced laminate is subjected to a uniform tensile stress. Albeit the results obtained compare qualitatively well to the experimental observations, no further details about the mechanical response and reliability of the mesh at this scale is presented. Ref. [19] analyzes the debonding between fibers and the surrounding polymer matrix in long fiber reinforced composites. In this two-dimensional configuration, the fiber-matrix interface is integrated into the XFEM scheme by using the Level-Set method and a cohesive interaction law is only considered for the enriched nodes of the interface. Ref. [20] addresses a two-dimensional problem to understand the fracture behavior of asphalt mixtures taking into account the interaction between their constituents and the matrix. This work describes the aggregates via XFEM embedded in a non-fracturable visco-elastic matrix, while the interface is represented using cohesive elements with finite thickness. Ref. [21] predicts the microcracking distributions and permeability in composite laminates. In this case, XFEM is used to describe the intra-laminar fracture process while the delamination between plies is represented by means of cohesive surfaces.

This numerical paper is devoted to present and describe in detail two well-defined examples in the context of encapsulation-based materials: concrete and polymer. In that sense, the influence of different aspects of the system design is numerically predicted (e.g., size, geometry, position and number of capsules). From the experimental point of view, to carry out an equivalent analysis would be unaffordable, and in part, what motivated to perform this work. Related to this, several detailed micro-mechanical aspects are presented, like for example the distribution of stresses between capsules or the range of bonding strength to capsule strength ratio to produce debonding or breakage. In terms of validation, the comparison of the proposed methodology with more simplified experimental setups is ongoing and it lays in the scope of a forthcoming work. It must be noted that, due to the numerical character of this paper, computational aspects like mesh convergence, parameter sensitivity or computational cost are thoroughly addressed, with the aim of providing reliable and feasible results for the next stage of validation.

This work is organized as follows. Section 2 presents a summary of the numerical approach that will be used in the following sections: the combination of XFEM and the CS technique implemented in the commercial software Abaqus. Details about the criteria regarding initiation and propagation of cracks and interface debonding are provided. Section 3 shows how this methodology can be applied to model in detail a realistic setup typically used to assess the encapsulation-based self-healing strategy in concrete structures. A numerical analysis to foresee the effect of capsules in the overall mechanical strength is provided and discussed. Section 4 addresses a similar study but oriented to a quite different material system and configuration, namely, encapsulation-based self-healing polymer materials. Unlike the previous section, here a micro-scale model is presented, where the interaction of a polymeric capsule with the surrounding polymer matrix is fully analyzed under periodic boundary conditions. This configuration makes it possible to assess in an efficient way the effect of important design variables like the capsule volume fraction. The prediction of the homogenized mechanical properties is presented and discussed in terms of the information available in the literature. Section 5 is devoted to establishing the limitations found so far during the execution of this approach using the commercial software to carry out this work. This paper finishes with Section 6 providing a summary of the main findings and some remarks about the applicability of the results.

\section{Modeling framework}

The simulations performed in this work rely on the combination of two computational techniques. On the one hand, the XFEM has been used to model the initiation and propagation of a crack that might be initiated both in the matrix and in the capsule. On the other hand, these two parts are attached to each other by means of the corresponding interface. The interaction between both surfaces is implemented via a surface-based cohesive behavior governed by a traction-separation law. Although a more specific description of these techniques can be found in ref. [22], for the sake of clarity, both techniques are summarized in the following paragraphs.

\subsection{XFEM-based cohesive segments to model solids}

This work uses a built-in implementation available in the commercial software Abaqus, where the cracks do not have to be prescribed and therefore, the initial solid structure is intact. Two criteria must be defined: the initiation of a crack and the propagation of such crack. This 
procedure relies on the explicit determination of the crack faces, whose locations are used to establish an interaction law between both lips which is dependent on the magnitude of their separation. Mesh discontinuities representing cracks are carried out with a combined strategy using the phantom node technique $[23,24]$ and the Level Set method $[25,26]$. Both procedures prevent the need of including the singular-dependence of the stresses around the crack-tip and the need to make the crack paths independent of the geometrical features of the mesh. A crack can appear in the centroid of any element of the mesh when the maximum principal stress calculated in its integration points satisfies the following criterion

$\max \left\{0, \frac{\sigma_{\text {maxps }}}{\sigma^{*}}\right\} \geq 1$

where $\sigma_{\operatorname{maxps}}$ is the calculated maximum principal stress and $\sigma^{*}$ stands for the maximum strength of the material. The adopted criterion indicates that a negative principal stress does not have any effect on the fracture response: the element cannot be fractured if it undergoes a compressive stress that exceeds the material strength $\sigma^{*}$. Therefore, this situation implies two things: (i) the physical meaning of $\sigma^{*}$ is the maximum tensile strength of the material, and (ii) Eq. (1) cannot be used as the only fracture criterion if crushing mode is also a dominant failure mode in the material. The extension of an already started (or prescribed) crack will occur if the element ahead of such crack fulfills Eq. (1), in which case the crack length will increase with a new segment cutting it.

After satisfying Eq. (1) in a certain element, a discontinuity oriented orthogonally to the maximum principal direction is introduced in that element. This discontinuity is represented by means of a jump in the nodal displacements via the phantom node method [23], which consists of adding an extra superposed node to every mesh node. When a mesh element is pristine, the phantom node overlaps and follows the movement of the real node. However, if an element is decided to be broken, the phantom nodes bounding this element are activated and their movements are decoupled from the real nodes. This extra set of nodes allows for unfolding the field of displacements of the original element into two parts. The physical location of the crack lips is determined using the Level Set method, which makes use of two signed distance functions to locate the material points above or below the crack surface, as well as those points laying ahead or behind the crack front. Full details can be found in refs. [25-28]. The amount of separation between a real node and its corresponding phantom node is ruled by a cohesivelike law. Both nodes become fully independent once the relative distance between them exceeds the cohesive threshold.

The aforementioned cohesive law, the so-called damage evolution law, controls the degree of softening, or cohesive stiffness, when the crack opens. Immediately after the insertion of the crack, the cohesive

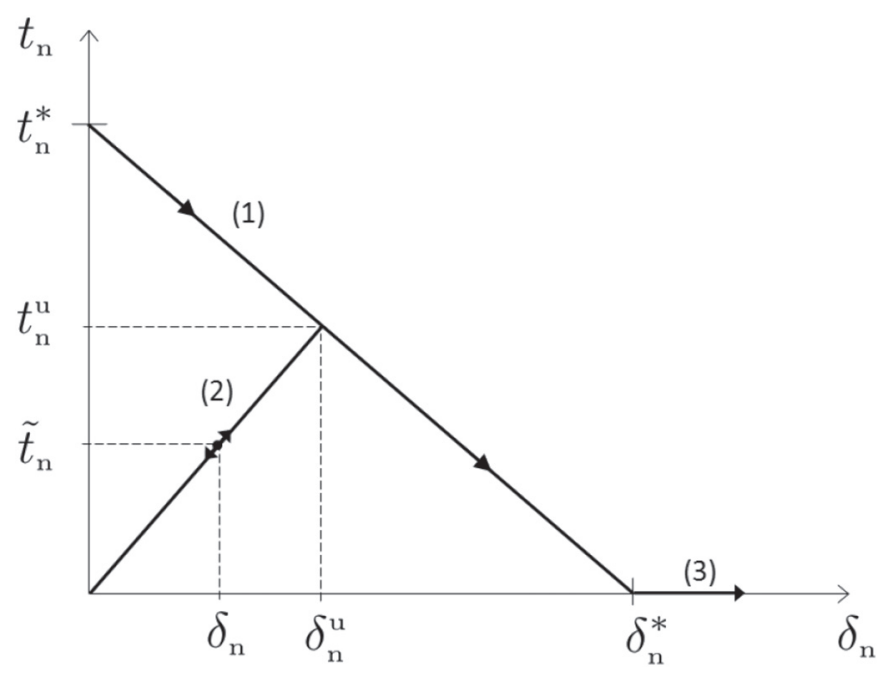

Fig. 1. Traction-separation response at the crack faces. traction between the crack lips is set equal to the material strength $\left(\sigma^{*}\right)$. Abaqus uses an "extrinsic" approach [29], which means that the evolution law is formulated independently from the initiation criterion, because the crack location is not known a priori. For the sake of conciseness, only the damage evolution of the normal component to the crack faces is described next.

Fig. 1 shows the traction-separation response in the normal direction to the crack faces. Three paths are identified: path (1) corresponds to the crack opening associated to a monotonic increase between the crack faces, path (2) represents the traction between the crack faces when either the crack arrests or it is newly being re-opened, and path (3) indicates the full fracture or traction-free state. This response can be summarized as follows

$\begin{array}{lll}\text { Path (1): } & t_{n}=t_{n}^{*}\left(1-\frac{\delta_{n}}{\delta_{n}^{*}}\right) & 0 \leq \delta_{n} \leq \delta_{n}^{*} \\ \text { Path (2): } & \tilde{t}_{n}=t_{n}^{*} \frac{\delta_{n}}{\delta_{n}^{u}}\left(1-\frac{\delta_{n}^{u}}{\delta_{n}^{*}}\right) & 0 \leq \delta_{n} \leq \delta_{n}^{u} \\ \text { Path (3): } & t_{n}=0 & \delta_{n} \geq \delta_{n}^{*}\end{array}$

where $t_{n}$ is the normal traction acting between both crack faces, $t_{n}^{*}$ is the maximum allowable stress at fracture initiation, $\tilde{t}_{n}$ stands for the unloading value of the normal traction, $\delta_{n}$ is the current normal distance between the crack faces, $\delta_{n}^{*}$ is the length of the cohesive interaction and $\delta_{n}^{u}$ indicates the crack opening just before unloading. According to Fig. 1 , the traction between the crack faces given by $\widetilde{t}_{n}$ experiences a degradation process that increases as the opening distance increases as well. This degradation during unloading is driven by

$\tilde{t}_{n}=\left\{\begin{array}{lll}(1-d) t_{n} & \text { if } \quad t_{n} \geq 0 \\ t_{n} & \text { if } \quad t_{n}<0 \text { (compression) }\end{array}\right.$

where $d$ is a scalar and monotonically increasing variable expressed as

$d=1-\frac{\delta_{n}}{\delta_{n}^{u}}\left(\frac{\delta_{n}^{*}-\delta_{n}^{u}}{\delta_{n}^{*}-\delta_{n}}\right)$

that quantifies, between 0 and 1 , the deterioration of the cohesive interaction. This degradation is necessary to account for the energy dissipated during the process of fracture. If no degradation was included, after unloading, the traction values would come back through the path (1), what would indicate an unrealistic elastic recuperation. Having said this, the area under this traction-separation law provides directly the fracture energy in mode-I. In this paper, due to the loading conditions that will be presented later on, this fracture mode is the most relevant to describe the material fracture. This degradation applies similarly to the shear stress components, in which case it reads as $\tilde{t}_{k}=(1-d) t_{k}$, with $k=s, t$ where $s$ and $t$ stand for shear and tangential components, respectively. For these cases, in case of negative shear and tangential stresses, an anti-symmetric triangle as the one shown in Fig. 1 is applied. More details can be found in ref. [22,29,30].

\subsection{Surface-based cohesive technique to model interfaces}

The capsules embedded in the matrix pre-define the interface. In this case, an "intrinsic" formulation for the traction-separation law between capsules and matrix can be used [30-32]. Unlike the treatment for XFEM above, both the initiation and propagation criteria are integrated in the same formulation. The capsule-matrix interface consists of a zero-thickness region that involves only the surface pairs initially in contact, as it is shown in Fig. 2 (left).

Independently of the capsule shape, the interaction that connects the external surface of the capsule with the matrix is modeled using the intrinsic traction-separation law showed in Fig. 2 (right). Again, for the sake of conciseness, this figure shows only the normal component of the interface stress. The two shear components parallel to the interface retain similar shape, except for having an antisymmetric shape with respect to the origin of coordinates $(\delta, T)$. Similarly to Section 2.1 , the normal interface stress from Fig. 2 (right) can be expressed as follows 

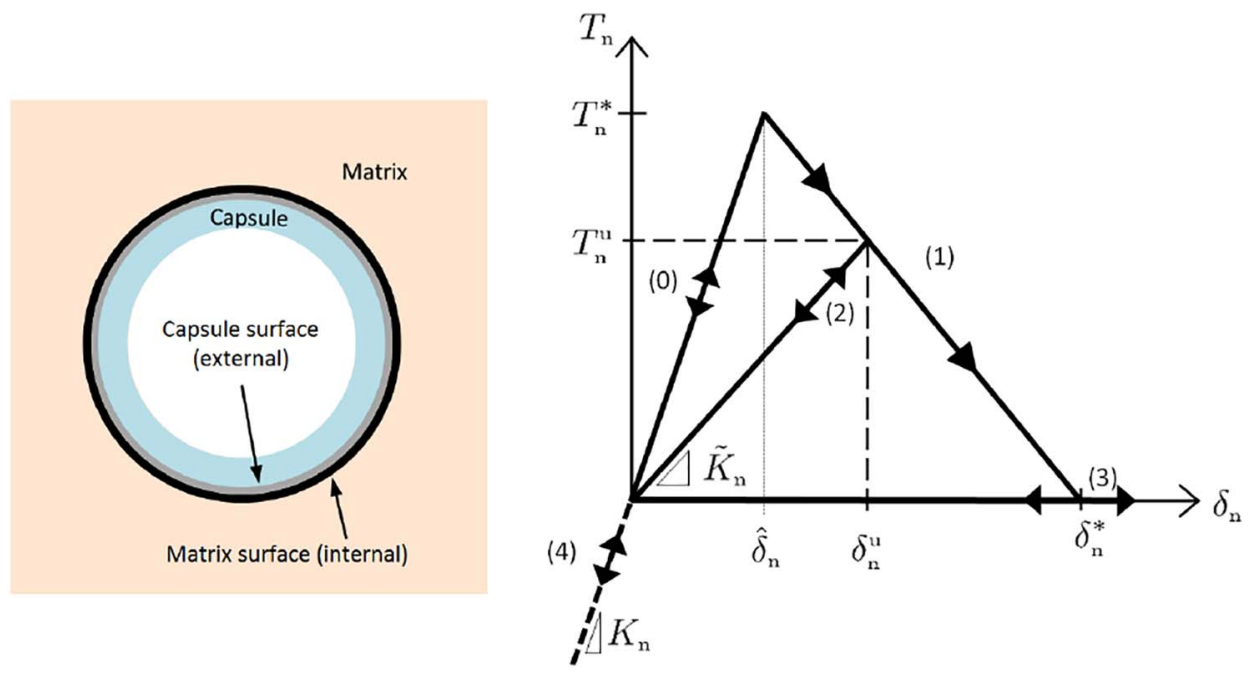

Fig. 2. Traction-separation response at the interacting surfaces of the capsule-matrix interface.

\begin{tabular}{|c|c|c|}
\hline Path (0), (4): & $T_{n}=K_{n} \delta_{n}$ & $\delta_{n} \leq \widehat{\delta}_{n}$ \\
\hline Path (1): & $T_{n}=T_{n}^{*}\left(\frac{\delta_{n}^{*}-\delta_{n}}{\delta_{n}^{*}-\hat{\delta}_{n}}\right)$ & $\widehat{\delta}_{n} \leq \delta_{n} \leq \delta_{n}^{*}$ \\
\hline Path (2): & $\widetilde{T}_{n}=\widetilde{K}_{n} \delta_{n}$ & $0 \leq \delta_{n} \leq \delta_{n}^{u}$ \\
\hline Path (3): & $T_{n}=0$ & $\delta_{n} \geq \delta_{n}^{*}$ \\
\hline
\end{tabular}

where $T_{n}^{*}$ is the maximum normal tensile stress allowable by the interface and $\widetilde{T}_{n}$ represents the degraded tensile stress when the interface is unloaded/reloaded. It must be noted that, unlike in the extrinsic approach, here the surface is initially stress-free, and a linear and elastic response, given by Path (0), is produced until reaching the critical value $T_{n}^{*}$. This elastic response is governed by the stiffness $K_{n}$, the so-called penalty stiffness, whose value is calculated as a function of the two adjacent material stiffnesses [31]. It has been proved that its value has no influence on the overall capsule-matrix stiffness [15]. Moreover, this work assumes that the normal and shear penalty stiffnesses are decoupled, therefore a pure normal opening of the interface does not produce shear forces, and vice versa. Once the interface reaches the peak stress $T_{n}^{*}$, or equivalently, the interface surfaces separate a distance $\widehat{\delta}_{n}$, the initiation of debonding is triggered. More generally, the initiation criterion is fulfilled when the following relation is met

$\max \left\{\max \left\{0, \frac{T_{n}}{T_{n}^{*}}\right\}, \frac{\left|T_{s}\right|}{T_{s}^{*}}, \frac{\left|T_{t}\right|}{T_{t}^{*}}\right\}=1$

where the subscripts $n, s, t$ stand for the normal, shear and tangential components of the interfacial stress. The starred stresses are the maximum stresses withstood by the interface in the corresponding direction (i.e., interface property) and are defined positive. This condition prevents debonding initiation when the interacting surfaces are only under pure normal compression.

If the aperture of the interface still continues, the process of weakening is followed through Path (1), until reaching the critical separation value $\delta_{n}^{*}$. At this state, within Path (3), the cohesive force between surfaces vanishes and the energy dissipated in the process corresponds to the interface fracture toughness (mode-I in this case showed in Fig. 2). Path (2) represents intermediate unload/reload of the interface after initiation and it yields the degradation given by

$\widetilde{K}_{n}=(1-D) K_{n}$,

where the damage variable of the interface is

$D=\frac{\delta_{n}^{*}}{\delta_{n}^{u}}\left(\frac{\delta_{n}^{u}-\widehat{\delta}_{n}}{\delta_{n}^{*}-\widehat{\delta}_{n}}\right)$.

If the interface undergoes compression, the pressure overclosure relationship governs the normal reaction, where the original penalty stiffness $K_{n}$ is used to prevent the interpenetration between both surfaces, as indicated by Path (4). It must be noted that Path (4) can also be reached even when the Path (3) has been reached in a previous stage; in this case both interface surfaces are treated as two noncohesive surfaces ruled only by the pressure overclosure relationship to avoid the aforementioned surface overlapping. Regarding relative tangential displacement (shear), when the interface is under compression but the shear cohesive stiffness is not damaged yet, the cohesive attraction parallel to the surface is activated. If any of the two shear stiffnesses reach the shear debonding initiation (see Eq. (6)), the degradation process will occur similarly as shown in Fig. 2. Furthermore, if full shear debonding takes place under compression, a frictional model can be considered. However, in the present paper, for the sake of simplicity a frictionless contact is assumed.

The area below the curve in Fig. 2 provides the energy dissipated to produce a new pair of fully disconnected surfaces, namely, the interface fracture toughness. The positioning of the capsules with respect to the applied load can generate mixed-mode propagation response of the cohesive interface. The propagation mode mixity involves different energies associated to the debonding capability in normal $(n)$ and parallel directions to the interface $(s, t)$. This work assumes that the interaction between the energies of each individual mode (i.e., $n, s, t$ ) follows the Benzeggagh-Kenane [33] model given by

$G^{*}=G_{n}^{*}+\left(G_{S}^{*}-G_{n}^{*}\right)\left(\frac{G_{S}}{G_{n}+G_{S}}\right)^{\eta}$

where $G^{*}$ is the total interface fracture energy, $G_{n}$ is the energy release rate calculated from the traction and normal displacement during interface opening, $G_{S}$ is the energy release rate calculated from the combined shear stress and its corresponding shear displacement (namely, $S$ collapses both $s$ and $t$ directions), and the power $\eta$ is a cohesive property parameter that describes the interaction between modes. The properties $G_{n}^{*}$ and $G_{S}^{*}$ represent the critical interface toughness related to each direction separately. The debonding is completed and can evolve when the total energy release rate calculated is greater than or equal to $G^{*}$. For the sake of simplicity, this model assumes that the critical fracture toughness is equal in all directions. Also, taking into account the effect of this parameter on the response [34], a tentative value $\eta=1.45$ has been used [35].

It is worth mentioning that, apart from these assumptions, more elaborated criteria could have been used (e.g., ref. [34]). However, given the high level of brittleness of the interface present in cementitious-based materials (Section 3.3), they would produce negligible effects in the mechanical response. Once the debonding has been triggered by reaching the interface peak strength, the difference in resistance to debonding between the three directions is negligible compared to the effect driven by such peak value (the softening slope is in general very steep for the studied cases). On the other hand, the 


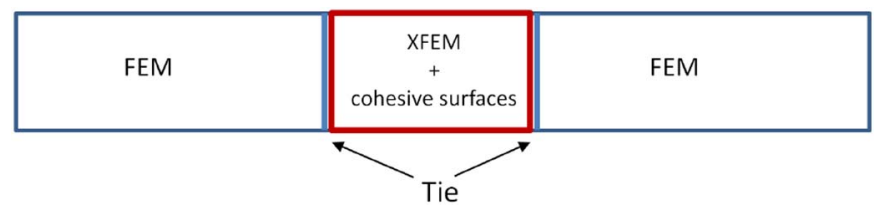

Fig. 3. Beam decomposition in three regions.

chemical affinity of the capsule and the matrix in polymer-based systems leads to quite strong interfaces. This situation rarely produces clear debonding, and therefore the breakage of the capsule becomes the main dominant mechanism. Anyway, both situations of capsule breakage and capsule debonding are equally studied in Section 4.1.

\section{Model application to self-healing concrete}

A common experimental setup used to assess the encapsulationbased self-healing strategy in concrete structures consists of using a classical three-point bending test [36-39]. Cylindrical capsules made of glass containing the healing agent are introduced in the mold with the help of a low-stiffness cord that sets their fixed position [39]. After positioning the capsules, the concrete is poured into the mold, the capsules being fully embedded in the concrete matrix. The choice of using glass capsules is mainly due to the brittleness matching of both materials at the scale size of the concrete constituents.

\subsection{Concrete beams with embedded tubular capsules}

This section presents a model that simulates the mechanical response of a realistic-sized notched concrete beam with several glass capsules embedded in the middle region of the beam, where a crack propagates till getting the total fracture of the beam. The aim of this sample model is to show how this methodology might be useful for experimentalists in order to get a better understanding of the interaction between cracks and capsules in a more realistic situation. Although the present example might be considered illustrative, the model developed for this work can easily be modified and used to explore different situations that affect the capsule breakage. These factors could be the number, size and positions of the capsules, as well as the possible mismatch of material properties (for example, quasi-brittle polymeric capsules), among others.

\subsection{Numerical setup}

This model consists of a rectangular beam composed by three regions: a central part where capsules are embedded and two wings. Fig. 3 shows schematically this composition.

The central part of the beam is numerically described by means of
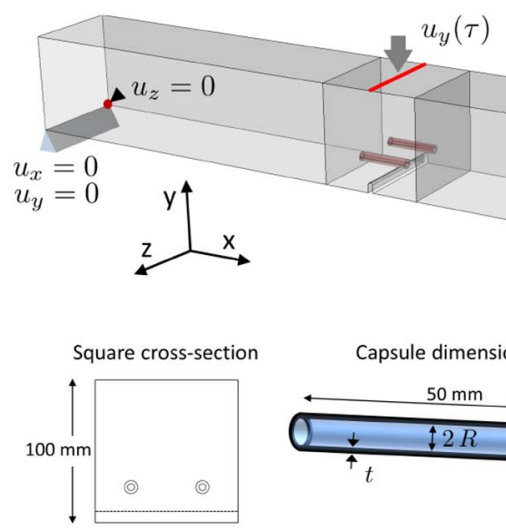

Capsule dimensions

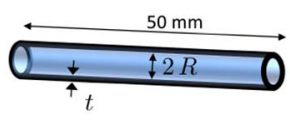

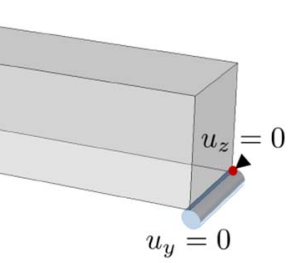

Notch dimensions

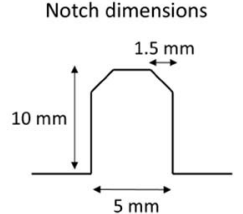

Fig. 4. (Top) Schematic view of the setup: geometry, global coordinate system, boundary conditions and prescribed load. (Bottom) Cross-section of the beam, capsules dimensions and geometry of the beam notch. the extended finite element method, whereas both lateral wings are described by using conventional finite elements. The central part contains the capsules at certain prescribed locations and similarly to this beam part, the capsules are also described by extended finite elements. Capsules are joined to the matrix by means of cohesive surfaces, as it will be shown later. The beam wings are connected to the central part via tie constraints. This tie is a surface-based ligament that makes it possible to join two different mesh types with different element density. The aim of this arrangement is to speed up calculations because the capsules have only been located in the neighborhood of the notch, as it is shown in Fig. 4. In this bending test, it is expected that a crack will emanate from the notch, and therefore, it will interact directly with the capsules located nearby. This makes it possible to study the role of the capsules in terms of effects of weakening or strengthening of the overall beam strength.

The beam is subjected to a three-point-bending load, where the span distance covers the whole beam length, as it is shown in Fig. 4 (top). This configuration simplifies the mesh details of the wings, but it can only be performed in a numerical test and not in a real specimen. Both support lines have restricted vertical movement whereas only one of them has restricted horizontal displacements along the axis- $x$. To prevent any rigid body motion, two rear nodes of the support lines are constrained along the the z-direction. The load is applied via a prescribed quasi-static vertical displacement on the center-top line of the beam as a linear function of time. This displacement at the instant $t$ is given by $u_{y}(\tau)=u_{y}^{\max } \tau / T$, where $\tau \in[0, T]$. All calculations performed in this work make use of the implicit solver of Abaqus/Standard, where the total duration of the load has been set $T=1$ and the maximum prescribed deflection was $u_{y}^{\max }=0.185 \mathrm{~mm}$.

The beam used in this work has a total length of $80 \mathrm{~cm}$ with a squared cross-section of $10 \mathrm{~cm}$, as it is shown in Fig. 4 (bottom). The width of the central region has been set to $10 \mathrm{~cm}$ and the wings length to $35 \mathrm{~cm}$ each one. All capsules used in this model have tubular geometry with a length of $50 \mathrm{~mm}$. For convenience, the capsule geometric ratio is defined by $t / R$, where $t$ is the wall-thickness and $R$ stands for the internal radius. The notch performed in the beam has been designed with a corner-beveled geometry, as it is shown in Fig. 4

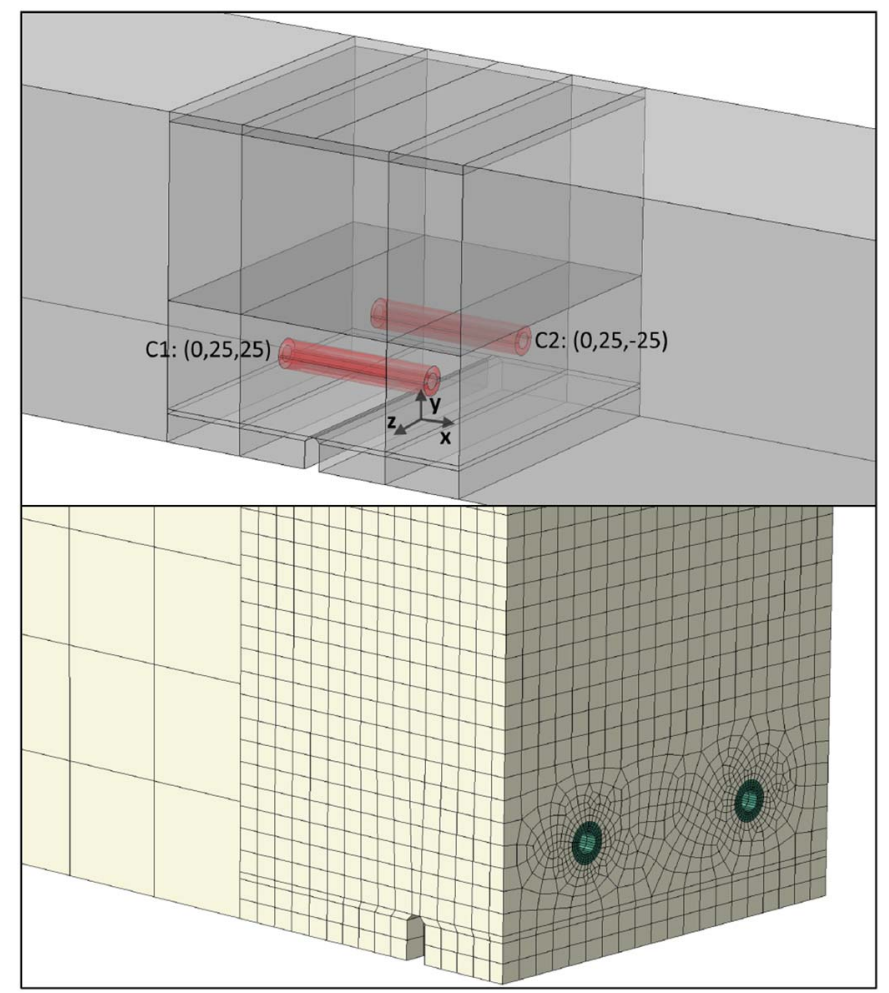

Fig. 5. (Top) Capsules location and coordinate system. (Bottom) Detail of cross-section of the mesh through the beam, using a maximum element size of $5 \mathrm{~mm}$ for the matrix. 
(bottom). This geometry captures relatively well the similarities to those notches produced after using a diamond saw in real concrete beams in the lab [3]. Moreover, the selected geometry enhances the regularity mesh with the minimum number of partitions. The height of the notch is set to $10 \mathrm{~mm}$ with a width of $5 \mathrm{~mm}$. Both dimensions correspond to the common notch size performed in real tests [39].

Regarding the insertion of capsules in the beam, a script written in Python programming language has been developed to be used in the Abaqus' command line interface (CLI) [22]. This script locates the capsules with prescribed user-defined coordinates, assuming that the local axis of reference is in the middle of the bottom surface of the beam, as it is shown in Fig. 5. Given the coordinates, the script creates automatically all the needed partitions in the central region according to the available space, in order to facilitate the subsequent meshing process (intermediate lines in the same top figure). In that sense, the necessary space required for the capsules is subtracted from the initially solid beam, and the seeds along the circumferential edges of capsules are adjusted to match those of the created tubular cavity in the beam with the aim of getting a circumferentially conformal mesh, as Fig. 5 shows. This procedure via scripting discards the formation of distorted elements and imposes a smoother gradient of element size from the beam element size up to the capsule element size.

The capsules and the central region of the beam have been discretized using 8-node linear brick elements with reduced-integration and hourglass-control (C3D8R in Abaqus nomenclature) [22]. The beam wings have been meshed using a fully regular grid of $25 \mathrm{~mm}$ length where 20-node quadratic brick elements have been used (C3D20), i.e. 224 elements per wing. Each capsule has uniformly been meshed a minimum of 4 elements through the thickness. In Section 3.4, devoted to the mesh effects, more details about element size employed for the beam and the capsules are presented.

\subsection{Material properties}

The elastic response of the materials used to represent the concrete and the capsules is isotropic, homogeneous and linear elastic, up to reach fracture. Therefore, their parameters are then represented by the Young's modulus, denoted by $E$, and the Poisson's ratio, denoted by $\nu$. The matrix elasticity is chosen to represent a typical concrete [40] and the elastic response of the glass is given by a conventional annealed glass [41,42]. Table 1 summarizes the values of material properties used in this work. The fracture properties are assumed to be those of quasi-brittle materials, according to the proposed model described in Section 2. The corresponding parameters are represented by the maximum tensile strength, $\sigma^{*}$, and the fracture energy, denoted by $G^{*}$. The subscripts " $\mathrm{m}$ ", "c" and "i" stand for matrix, capsule and interface, respectively.

There is a vast variety of concrete available depending on the application, however, a common range of the critical stress intensity factor for a typical unreinforced concrete under tension is $K_{I C}=0.9-1.5 \mathrm{MPa} \sqrt{\mathrm{m}}$ [43-45]. Unless stated otherwise, this work uses an intermediate value equal to $1.2 \mathrm{MPa} \sqrt{\mathrm{m}}$. In terms of fracture energy, the latter value corresponds to $G_{m}^{*}=36 \mathrm{~J} / \mathrm{m}^{2}$, by using the approximate relationship for stationary cracks $G_{m}^{*}=K_{I C}^{2} / E$ and the Young's modulus from Table 1. Similarly, the maximum tensile strength of unreinforced concrete can vary within the range $2-5 \mathrm{MPa}$ [40]. This work makes use of a conservative value given by $\sigma_{m}^{*}=2.5 \mathrm{MPa}$. Regarding the fracture parameters for glass, it is known that the tensile strength exhibits a very high variability even when specimens similarly

Table 1

Material properties: $E$ - Young modulus, $\nu$ - Poisson ratio, $\sigma^{*}$ - Tensile strength and $G^{*}$ Fracture toughness energy.

\begin{tabular}{lllll}
\hline & $E(\mathrm{GPa})$ & $\nu(-)$ & $\sigma^{*}(\mathrm{MPa})$ & $G^{*}\left(\mathrm{~J} / \mathrm{m}^{2}\right)$ \\
\hline Matrix $\left(_{m}\right)$ & 40 & 0.2 & 2.5 & 36,75 \\
Capsule $\left.{ }_{c}\right)$ & 70 & 0.2 & 60 & 8 \\
Interface $\left.{ }_{i}\right)$ & - & - & $1,2,5,10$ & 1 \\
\hline
\end{tabular}

produced are tested in the same conditions $[46,47]$. A common interval of failure stress ranges from 15 to $200 \mathrm{MPa}$, depending on whether some specific treatment is carried out in order to increase the mechanical performance [48]. For the purposes of this work, an intermediate value equal to $60 \mathrm{MPa}$ has been used. This value represents approximately the strength value between a conventional annealed glass and a heat-strengthened glass [47]. Similarly as for concrete, for reference, the fracture energy for typical soda-lime glass in ambient air ranges approximately from 6 to $9 \mathrm{~J} / \mathrm{m}^{2}[49,50]$. A value equal to $8 \mathrm{~J} / \mathrm{m}^{2}$ has been used in this work.

The maximum interface tensile strength $\left(\sigma_{i}^{*}\right)$ formed between concrete matrix and glass capsules, hereafter referred to as bonding strength, provides a measure of the static normal load required to separate a piece of concrete fully adhered to a flat glass surface. The particular values of this interface strength are vaguely reported in the literature, with the exception of some works [51,52]. In these works, a glass-concrete interface was created just placing a conventional glass plate in contact with fresh concrete, and allowing the bimaterial sample to cure as usually done with pieces only made of concrete. In that sense, no additive nor surface treatment to enhance the adherence between both materials was used. These studies showed that this interface is extremely brittle and the displacement involved to reach the interfacial failure lies in the order of one micron. Furthermore, they also obtained an average value of approximately $1 \mathrm{MPa}$, both debonding in mode-I and mode-II. In this work, with the aim of investigating the applicability of the proposed model, according to Table 1, a total of four values of $\sigma_{i}^{*}$ have been studied. The values equal to $1 \mathrm{MPa}$ and $2 \mathrm{MPa}$ might be considered as low-to-medium values of the capsule bonding strength. However, the values $5 \mathrm{MPa}$ and $10 \mathrm{MPa}$ might be assumed as strong interfacial values, in which case the surface would have been treated in order to improve the adhesive strength. On the other hand, the interfacial fracture toughness, denoted by $G_{i}^{*}$, provides a measure of the difficulty to extend an existing interfacial crack between the two aforementioned adhered surfaces. For all cases studied in this work, this value has been kept constant and equal to $1 \mathrm{~J} / \mathrm{m}^{2}$. This value generates a very brittle response typically found not only in glass-concrete interfaces but also in interfaces formed between glass substrates and different cementitious-based materials [17].

\subsection{Mesh convergence}

In order to establish the degree of mesh refinement required to obtain reliable results, several preliminary calculations using a notched beam without capsules have been studied. Five element sizes have been used to mesh the central region of the beam: 15, 12.3, 9.6, 7.0 and 5 $\mathrm{mm}$. As mentioned in Section 3.2, the element size of the beam wings has been kept constant to $25 \mathrm{~mm}$. It has been checked that using smaller elements to mesh the wings has a negligible effect on the global results, although it increases considerably the computational time. Overall, all element sizes here tested are well below the value ruled by the critical element size discussed in reference [53], and whose value is of the order of half a meter for the concrete properties from Table 1. However, given the size of the specimen, an adequate mesh size has to be found in order to guarantee mesh independence in terms of size, as XFEM is able to deal with the true discontinuous character of cracks [54], unlike in a continuous damage approach [55].

Fig. 6 shows the force-displacement curves for two sets of simulations. The first set, with curves in black color, corresponds to the concrete properties defined as the reference properties $\left(\sigma_{m}^{*}=2.5 \mathrm{MPa}\right.$, $G_{m}^{*}=36 \mathrm{~J} / \mathrm{m}^{2}$ ). The second set was performed using a concrete material with the same tensile strength but setting a higher fracture toughness $\left(G_{m}^{*}=75 \mathrm{~J} / \mathrm{m}^{2}\right.$, curves in grey color). Due to this higher toughness, the total imposed displacement was increased up to $0.24 \mathrm{~mm}$, which is enough deflection as to make the crack front pass clearly the neutral axis of the beam. The aim of this analysis is to check whether the element size chosen for the reference properties is also valid in case of dealing with a tougher concrete. As it can be appreciated, using an element size below $10 \mathrm{~mm}$ already produces a very similar response, despite some small differences at the end of the load. This last part 


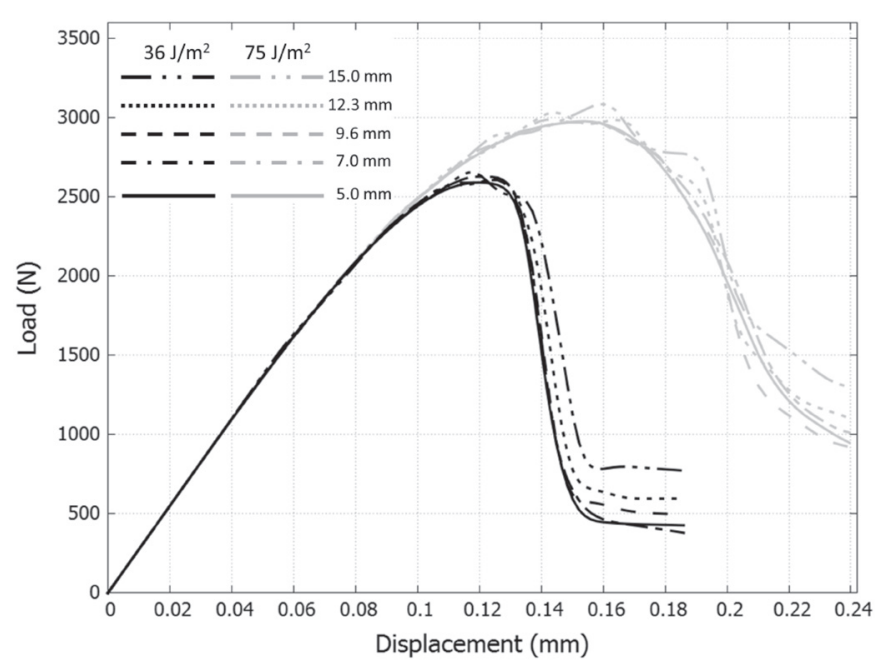

Fig. 6. Load-displacement curves for different element sizes.

corresponds to the moment when the crack has propagated more than half beam thickness. This toughening region is very sensitive to the specific trajectory followed by the crack tip. In that sense, any small deviation can activate a more expensive propagation mode than mode-I in bending. This situation can increase the force required to deflect the beam. Under a pure mode-I at the crack tip, like in the present case, the crack trajectory should be a plane perpendicular to the beam axis. However, as it will be shown in Section 5.1, the current XFEM implementation can introduce artifacts that are originated by a still improvable interaction of the crack tip and the boundary between mesh elements. It will be shown that as long as the crack lips intersect cleanly the elements, the crack pattern seems to be physically plausible.

Taking into account the computational cost of these simulations, as Fig. 7 shows, for practical purposes an element size equal to $7.0 \mathrm{~mm}$ has been used. As it can be seen, using a smaller size does not increase the resolution of the response but increases the computational time drastically. These analyses were carried out in a workstation with a processor Intel Xeon E5-2667 2.9 GHz. The maximum amount of memory used by the finest mesh was over 2.5 Gbytes. This is an important practical feature because the introduction of capsules necessarily enforces to use a finer mesh around and in the capsule itself, as it is shown in Fig. 5.

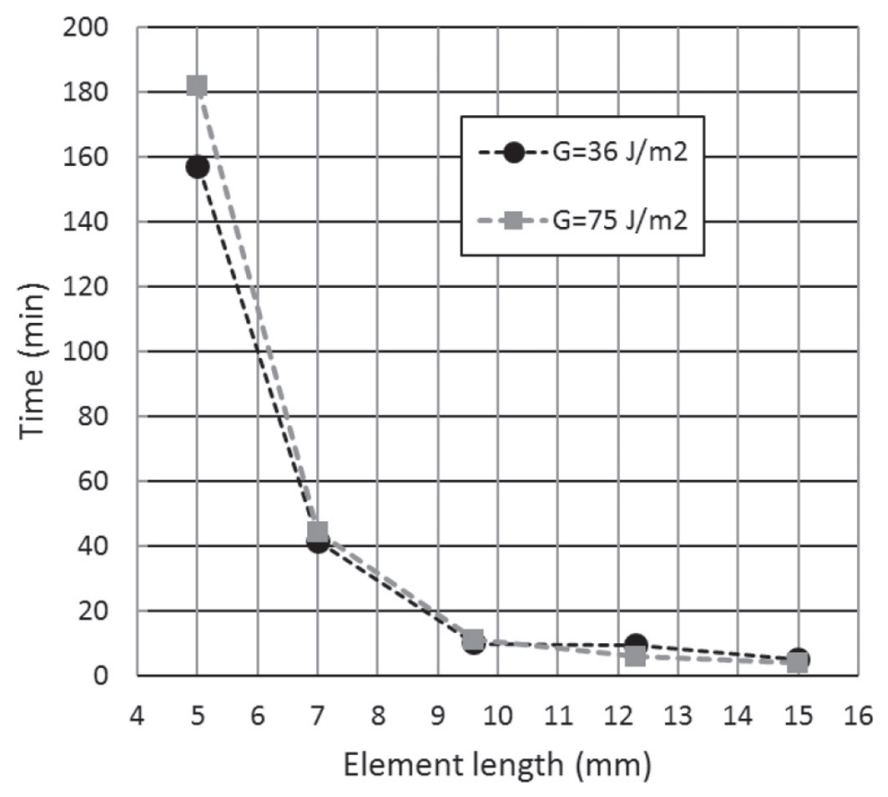

Fig. 7. Computational time to simulate fracture of a plain unreinforced concrete beam as a function of the element size.
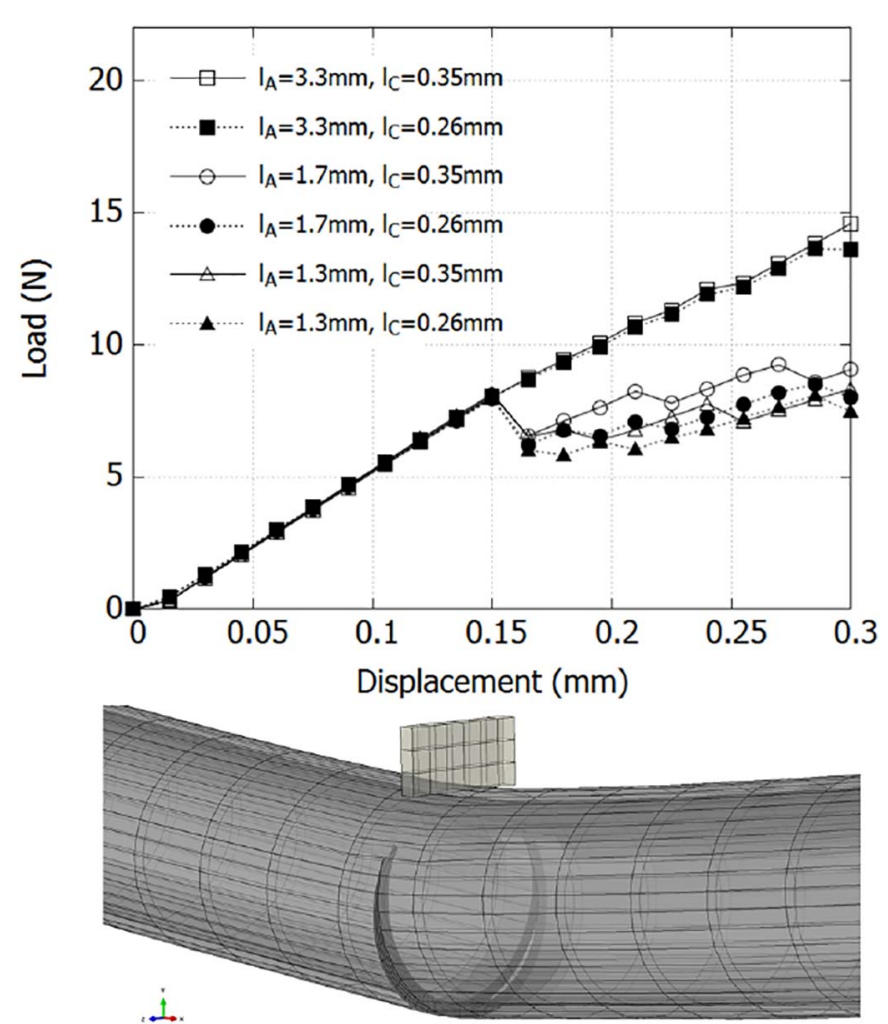

Fig. 8. (Top) Load-displacement curves for different element sizes used to mesh the capsule. (Bottom) Aspect of the capsule breakage under a bending deflection equal to $0.3 \mathrm{~mm}$.

A similar analysis has been performed to identify the optimal element size for the glass capsules. Fig. 8 shows the results when a single capsule was tested under three-point bending load. The capsule has an internal radius of $1.5 \mathrm{~mm}$ and thickness of $0.175 \mathrm{~mm}$. The capsule is simply supported at the lowest points of each side, with a span equals to its length, $50 \mathrm{~mm}$. The load is applied at the middle of the top part using a thin rigid stub that imposes a total displacement of $0.3 \mathrm{~mm}$ (see snapshot of Fig. 8). The capsule is not precracked, and it is expected to initiate a crack due to bending at the middle of the bottom part. This loading condition is not equivalent to the real one (i.e., embedded capsule that undergoes a combination of bending and uniform traction), nevertheless, from the numerical point of view, this test is equally useful to assess the mesh influence. Two mesh lengths have been distinguished: $l_{A}$ is the length of the element along the capsule axis and $l_{C}$ stands for the length of the element used to discretize the circumferential contour. The number of elements through the thickness was fixed to 4 (that corresponds to a radial element length of $44 \mu \mathrm{m}$ ). The sizes $l_{A}$ and $l_{C}$ are the critical ones because they might be bigger than the recommended critical element size for glass, which is about $0.3 \mathrm{~mm}$. Unfortunately, meshing with such a small element for capsules would generate a huge final mesh for the whole problem (beam with capsules) and it would become computationally unaffordable. Therefore, several larger element sizes have been tried, with the aim of determining which size can generate a reproducible and characteristic mechanical response. Fig. 8 (top) shows the forcedisplacement response for several combinations of $l_{A}$ and $l_{C}$. The expected response consists of three stages: (i) a linear response corresponding to the bending load before failure, (ii) the initiation of a crack, revealed by an interruption of the previous linear regime via a force drop and (iii) crack propagation until the final fracture. It is worth mentioning that a deflection of $0.3 \mathrm{~mm}$ was not enough to break the capsule completely, as it is shown in Fig. 8 (bottom). In any case, this level of maximum deformation was enough for the purposes of this analysis. In that sense, it has been obtained that $l_{A}$ is the most critical length, in such a way that using a value larger than $2 \mathrm{~mm}$ produces 
visible deviation of the expected response. However, using a circumferential length of $0.35 \mathrm{~mm}$ or $0.26 \mathrm{~mm}$ produced small differences. Therefore, the pair of values given by $l_{A}=1.7 \mathrm{~mm}$ ( 30 axial elements) and $l_{C}=0.26 \mathrm{~mm}$ (a minimum of 40 circumferential elements) were used, as they represent an acceptable compromise between accuracy and computational effort. Regarding the interface, taking into account the elasticity of the two adjacent materials (concrete and glass) and the low level of bonding strength, it has been checked that the selected meshes behave consistently with what has been reported by other authors [31,56].

\subsection{Interface effect on bending response}

This section presents the mechanical response under bending of the beam shown in Fig. 4. This beam contains two capsules, and they are inserted with a distance of $25 \mathrm{~mm}$ above the notch.

By using the proposed model, two different, but related, mechanical aspects are studied: on the one hand, the role of the insertion of capsules in the reinforcement or weakening effect of the overall beam strength under bending, and on the other hand, the analysis of which interface strength and capsule size are more beneficial to make the capsules break properly and, in this way, carry out the leaking of the healing agent into the crack. Regarding the latest aspect, this work is restricted to analyze the breakage of the capsules, and therefore they are assumed empty.

It is worth mentioning that in the case studied, the beam has a central notch to constrain the crack path along its middle plane with the aim of enforcing the intersection with the capsules. This is a common procedure used in lab tests $[3,57,58]$. Therefore, the path will not be affected by the material properties as it might occur if the absence of symmetries was constraining the problem [59].

Four values of the capsule geometric ratio were studied: $t / R=0.12$, $0.23,0.46,0.8$, being the pairs $(t, R)=(0.175,1.5),(0.4,1.75),(0.92$, 2 ) and $(2,2.5)$, respectively (in $\mathrm{mm}$ ). According to Table 1 , also four values of the maximum interface tensile strength $\left(\sigma_{i}^{*}\right)$ were used for each geometric ratio. Therefore, these combinations of capsule size and interface strength represented a total of 16 simulations.

Fig. 9 shows the response of the load as a function of the imposed displacement for three representative cases of bonding strength and capsular geometric ratio. In this plot, the case $\sigma_{i}^{*}=2 \mathrm{MPa}$ has been omitted because it produced a similar global response to the case $\sigma_{i}^{*}=1$ MPa. Similarly, for the sake of clarity, the case $t / R=0.23$ has also been omitted in this figure. For reference, the result obtained from a concrete beam without capsules (labeled "Solid", gray line) has also been included in order to facilitate the comparison when the capsules are present. As expected, the insertion of only two capsules has no effect on the global elastic response, because the capsule volume fraction is still very small compared to the volume fraction occupied by the concrete

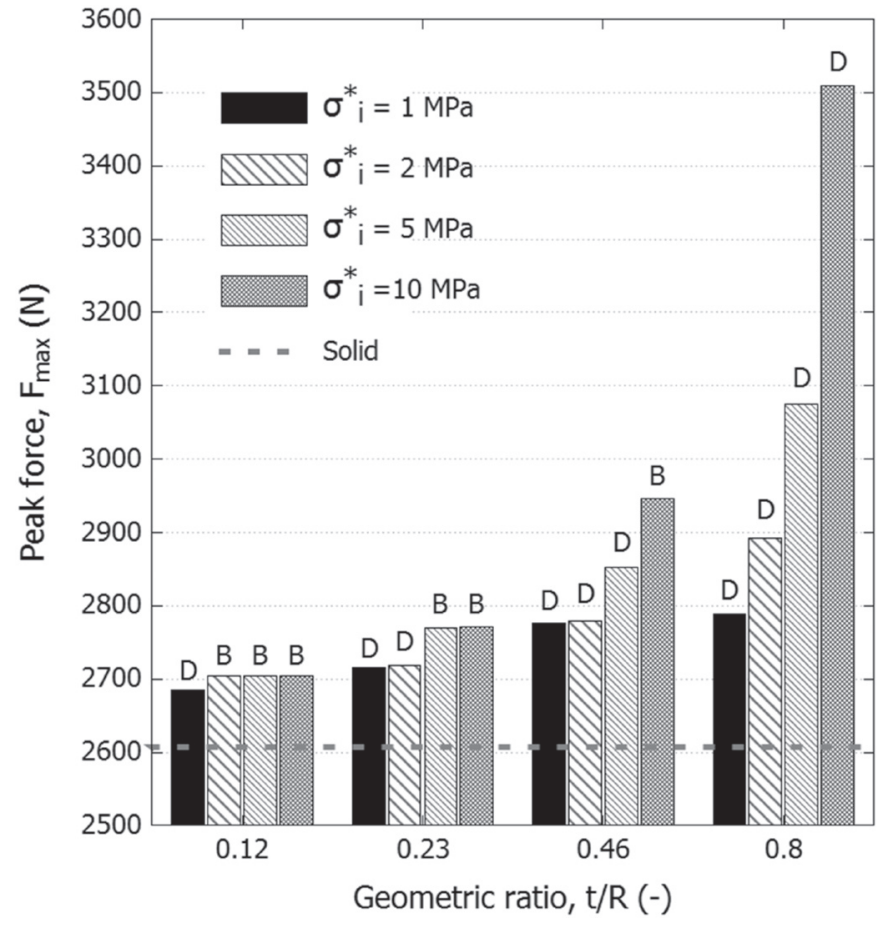

Fig. 10. Maximum force developed by the beam as a function of the geometric ratio and different interface strength levels. ( $\mathrm{D}=$ debonding, $\mathrm{B}=$ breakage).

material $(\approx 99.92 \%$ for the case $t / R=0.8$ ). Fig. 9 shows that the insertion of two small capsules $(t / R=0.12)$ contributes very poorly to the mechanical response, even though a strong bonding interface is used. In any case, it is expected that capsules with low cross-sectional geometric ratio (either the capsules detach or break), have a poor effect on the overall response under bending, mainly due to the weak efforts required to damage them. However, this situation changes when thicker capsules are used, as it will be shown later on. Nevertheless, due to practical reasons, such small capsule size is of great interest for experimentalists in self-healing and therefore, the effect of the number of capsules has been treated in more detail in the next Section 3.8. Also, as general feature from all cases, the presence of the capsule seems to make the shape of the curve sharper, being this a consequence of the high degree of brittleness of the capsule as well as the low value of the interface fracture energy (see Table 1). Fig. 9 clearly shows the important effect of the geometric ratio: when the capsules are better
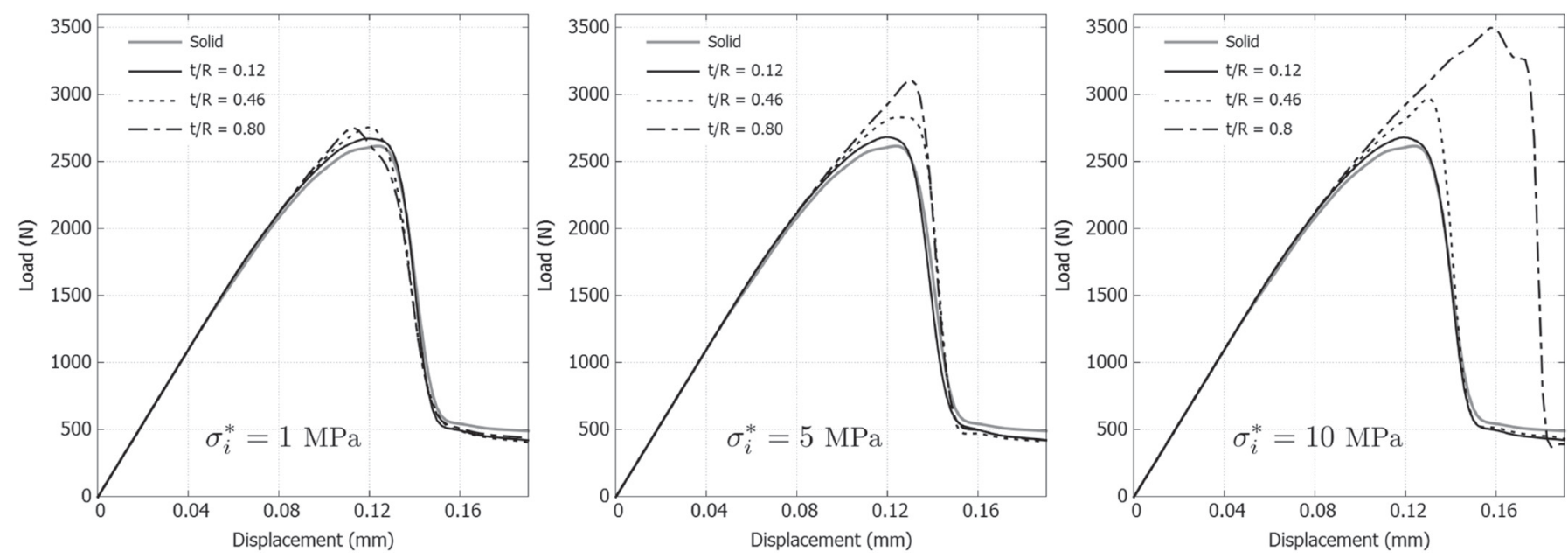

Fig. 9. Load-displacement curves for different capsule-matrix interface strength $\left(\sigma_{i}^{*}=1,5,10 \mathrm{MPa}\right)$ and geometric ratios of the capsule $(t / R=0.12,0.46,0.8)$. 
bonded to the matrix, the maximum force developed under bending also increases, although this increase, in a non-linear fashion somehow, becomes more evident when thicker capsules are employed.

\subsection{Capsule size effect}

Fig. 10 presents the maximum force extracted from the loaddisplacement curve as a function of the geometric ratio. In this plot, the dotted gray line establishes the baseline in strength calculated from the beam without capsules (Solid).

As it can be appreciated, albeit less visible in the previous Fig. 9, the insertion of two small thin capsules $(t / R=0.12)$ already leads to a moderate increase of the maximum load that the beam can resist under bending. In terms of reinforcement effects, when the bonding strength equals $1 \mathrm{MPa}$, Fig. 10 shows that an increase of the geometric ratio has a moderate rise of the peak force, although the capsules debond (labeled as "D"). This slight rise of load (for the thickest capsule up to $\sim 200 \mathrm{~N}$ ) can be due to the amount of surface initially in contact with the matrix: a capsule with larger external diameter has a larger area bonded to the matrix, and therefore, the force required to get separated from the concrete is also higher (this surface is almost 3 times greater for $t / R=0.8$ compared to the case $t / R=0.12$ ). When the interface strength increases to $2 \mathrm{MPa}$, the effect of the geometric ratio leads to a somewhat more visible effect on the peak force compared to the previous case $\sigma_{i}^{*}=1 \mathrm{MPa}$. Moreover, this level of bonding is already sufficient to break the capsules with a geometric ratio $t / R=0.12$ (labeled as "B", in Fig. 10). As mentioned before, this capsule size is commonly used by experimentalists to test encapsulation-based selfhealing concrete, because sufficient amount of healing agent can be introduced and dispersed by the concrete structure. Therefore, in terms of achieving an adequate healing mechanism, this result suggests that, as least, an interface strength of $2 \mathrm{MPa}$ has to be ensured in order to break and liberate the fluid content inside the crack gap. Conversely, a weaker capsule-concrete interface would not be sufficient to break the capsule and, as a consequence, the healing agent would not be released.

Following this reasoning, if for some practical reason, or technical requirement, thicker capsules are used (for example, with the aim of strengthening the beam simultaneously), the current model makes it possible to quantify the strength level of the interface to trigger the fracture of the capsule under certain level of deflection. In this particular case, Fig. 10 shows that capsules with $t / R=0.23$ require an interface strength equal to $5 \mathrm{MPa}$ to break them, while doubling this value of $t / R$ requires also to double the value of $\sigma_{i}^{*}(t / R=0.46$ requires $10 \mathrm{MPa})$. However, with the insertion of much thicker capsules $(t /$ $R=0.8$ ), even $10 \mathrm{MPa}$ is not sufficient to break the capsules, although a considerable reinforcement is positively achieved.

\subsection{Detailed inspection of breakage mechanism}

As mentioned before, capsules with geometric ratio $t / R=0.12$ ( $t=0.175 \mathrm{~mm}, R=1.5 \mathrm{~mm}$ ) has been preferred for experimentalists to perform laboratory tests to analyze the healing efficiency of the encapsulated-based self-healing concrete. Therefore, this section provides a detailed analysis of the breakage sequence of the beam using this capsule size.

This analysis presents the differences of the global response when the capsules detach or break. Fig. 11 (a) shows the load-displacement curve for the cases $\sigma_{i}^{*}=1 \mathrm{MPa}$ and $\sigma_{i}^{*}=2 \mathrm{MPa}$. As it can be observed, although both cases produced a very similar response, in fact, the internal failure mechanism was different. As described before, these thin capsules detached from the matrix when the interface strength was $1 \mathrm{MPa}$, however, they fractured when the interface increased to 2 $\mathrm{MPa}$. The latter value seems to be high enough to transfer the stresses from the matrix to the capsule. The point $B$ marked in the solid curve in Fig. 11 (a) indicates the moment in which a crack initiates in the capsule. This crack can be appreciated in the zoomed region depicted in Fig. 11 (b). Conversely, when the interface is weaker, that is, $1 \mathrm{MPa}$, at that point the capsule initiates the debonding process: the interface cannot withstand the pull-out stress and the capsule slides into the cavity. The point labeled $D$ marked on the dotted curve in Fig. 11 (a) indicates the moment immediately after full debonding occurred. Likewise, a zoomed view of this state can be seen in Fig. 11 (b). In this case, the capsule lost contact in the right side while its left side still remained attached to the matrix. The points $B$ ' and $D^{\prime}$ correspond to two more advanced stages during the bending process, in which the crack has exceeded the neutral axis of the beam, and therefore, the overall bending load has dropped significantly. This result suggests that the distinction between the capsule failure and capsule debonding is almost impossible to detect from the macroscopic point of view.

\subsection{Effect of the number of capsules}

This section analyzes the impact of the number of capsules with $t /$ $R=0.12$ inserted in the beam. A total of seven cases have been simulated, from two capsules up to eight capsules. In all cases, the capsules were located always at the same vertical distance from the notch, as indicated in Fig. 5, and evenly distributed along the line perpendicular to the beam axis. Similarly, the interface tensile strength was set to $\sigma_{i}^{*}=1 \mathrm{MPa}$ for all cases.

Using this level of bonding strength, all capsules detached from the concrete matrix, as it can be seen in the snapshots shown in Fig. 12. This figure depicts two sample cases, the left one, a beam with 6 capsules and right one, a beam with 8 capsules. All cases studied showed that all the capsules contained in the beam debonded simultaneously, although not all of them debonded at the same side of the notch. The latter behavior might be originated by the solver itself, for example, the order in which the equations are solved or the numerical precision. In that sense, in a geometrically symmetric problem, small numerical differences introduced by the mesh procedure, for example, may trigger the initiation of debonding slightly sooner in one side than in the other, in spite of both sides having reached the critical debonding stress.

In terms of the force versus displacement curve, it is worth mentioning that the eight cases produced a very similar overall response, i.e. the same curve shape, looking all of them very similar to the curves already depicted in Fig. 9 (left) and Fig. 11 (a). However, some differences were recorded regarding the value of the maximum load reached during the bending process. With the aim of presenting more clearly these results, Fig. 13 shows the peak force extracted from the load-displacement curve for each case simulated. This plot has double y-axis: the left axis quantifies the total load experienced by the beam, while the right axis (with font in gray color) quantifies the average force contributed per capsule. This last number has been defined as $\left(F_{\max }-F_{\text {solid }}\right) / N_{c}$, where $F_{\max }$ is the peak force obtained under bending when $N_{c}$ capsules are inserted and $F_{\text {solid }}$ stands for the peak force obtained when the beam has no capsules (Solid case, see horizontal black dashed line in Fig. 13).

Focusing on the reinforcement effects by inserting glass capsules, these results not only show that increasing more capsules increases the overall tensile strength too, but this increase occurs in a linear way (see black squares). However, the force contribution per capsule decays as the number of them increases (see gray circles). This behavior might be explained by two counteracting events that are taking place simultaneously. On the one hand, the insertion of the capsules represents an increase of resistance to the external load required to break the concrete beam. This feature has been analyzed previously, and it happens regardless of whether the capsules debond or break. On the other hand, the gradual increase of capsules also leads to reduce progressively the distance between them. As a consequence, this situation facilitates the interaction of the stress fields generated by every capsule and therefore, modifies the level of stress of the matrix housed in between them.

Fig. 14 shows the profile of tensile stress ( $\sigma_{x x}$, parallel to x-axis) extracted from the segment that stands along the beam depth and passes through the center of the capsules (dotted line parallel to z-axis depicted in upper snapshot). Two sample cases are depicted and compared: a beam with 2 and 6 capsules, where the capsule interdistance are $50 \mathrm{~mm}$ and $14.38 \mathrm{~mm}$, respectively. The data in both cases were extracted at the same applied deflection $(0.07 \mathrm{~mm})$. At this stage, 


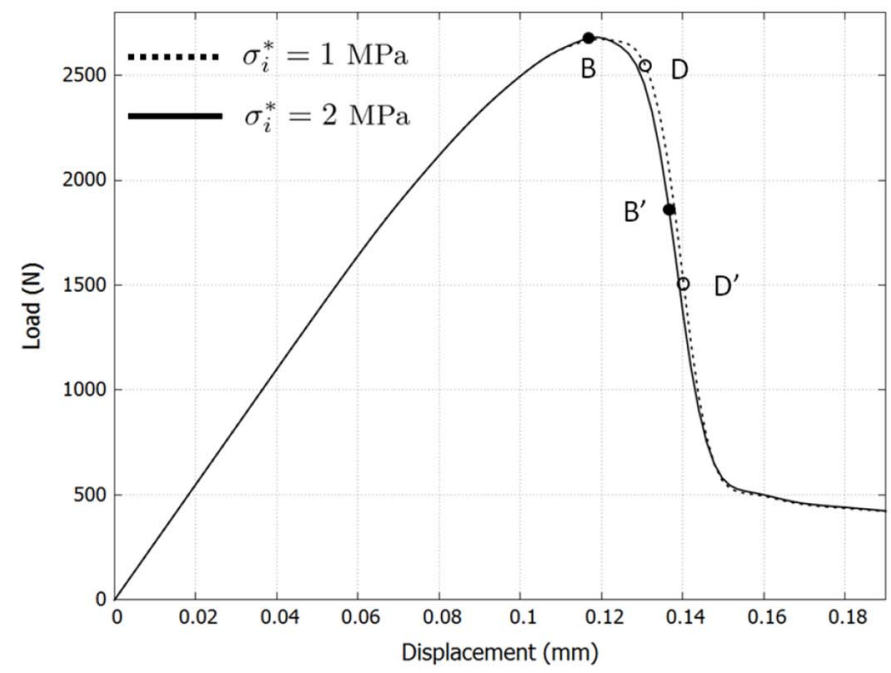

(a)
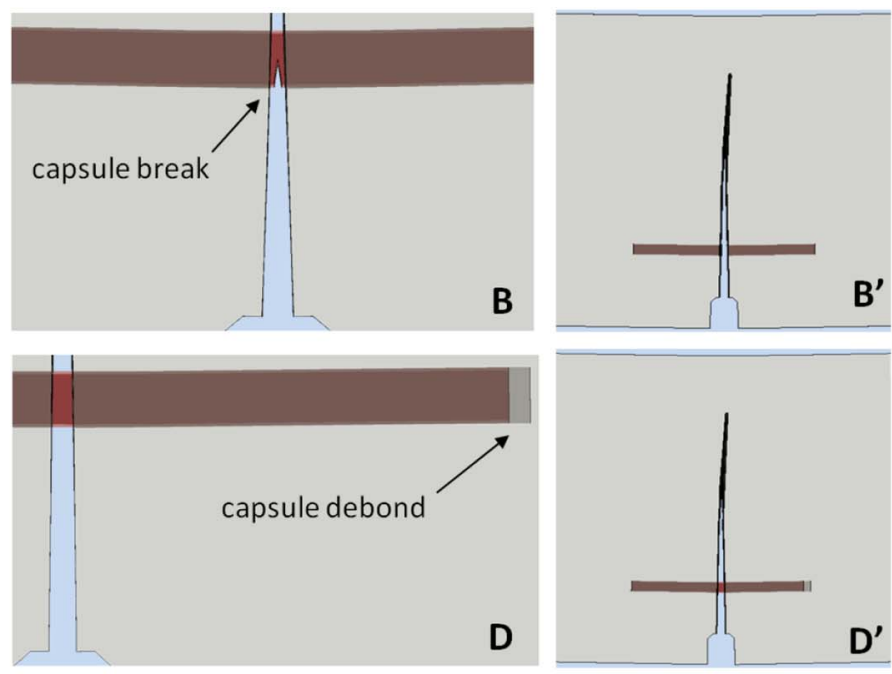

(b)

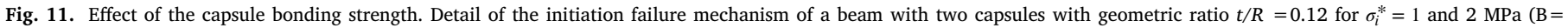
breakage, $\mathrm{D}=$ debonding). (a) Load-displacement curve. (b) Detail of the capsule breakage and debonding.
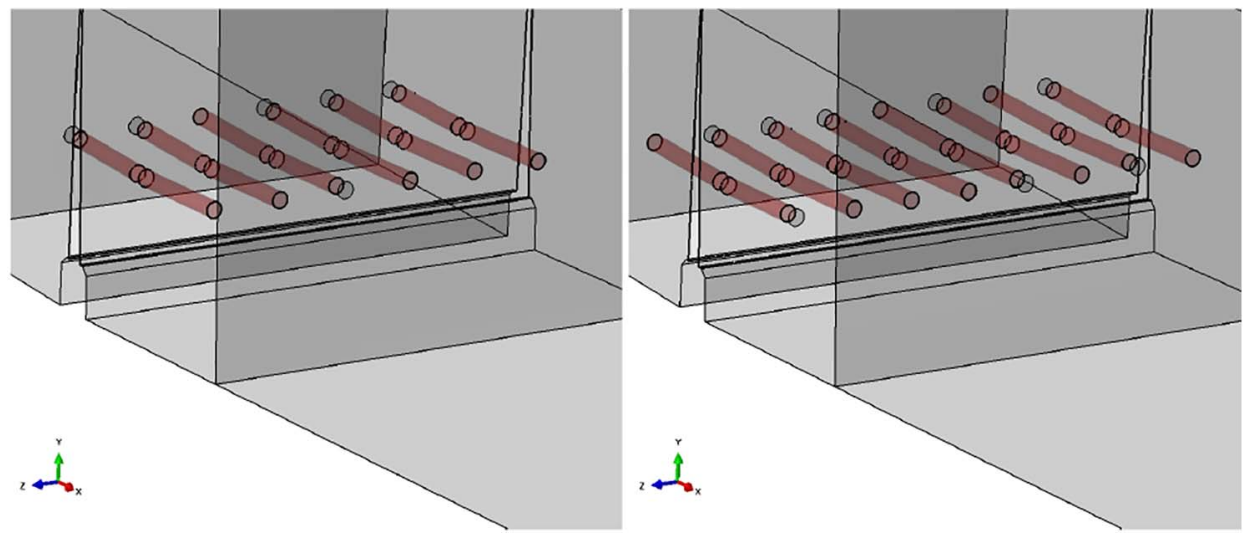

Fig. 12. Zoomed view of the crack path and debonding of capsules: (left) beam with 6 capsules and (right) beam with 8 capsules (deformation scale factor $\times 100$ ).

the crack front had not reached the capsules yet, as it can be observed in the top image of Fig. 14. At the same level of applied deflection, the tensile stress in the matrix is higher when the number of capsules increases, or equivalently, when the distance between capsules is shortened. In the regions where capsules are more densely clustered, the intermediate matrix is being more susceptible to fail sooner despite these capsules are working as reinforcement elements. Therefore, this result might suggest that capsules should be sufficiently separated from one another as to prevent their interaction, and therefore, to prevent a premature failure of the matrix in between them. In other words, a minimum capsular interdistance should be respected in order to get an optimal overall performance. Further analysis to quantify an optimal capsular clustering lies in the scope of forthcoming research.

\section{Model application to self-healing polymers}

Although there are several types of self-healing polymers [60], this modeling application is focused on polymers with embedded microcapsules which carry the healing agent. This type of self-healing polymers has been studied experimentally using the Tapered Double Cantilever Beam (TDCB) specimen via the so-called White protocol [61]. The experiments show how the cracks go through the polymer breaking the micro-capsules and, as consequence, delivering the healing agent in the crack plane.

\subsection{Polymer matrix with spherical micro-capsules}

Numerical simulations of the whole TDBC specimen with microcapsules are limited due to the difference of the dimensions between both the specimen, with dimensions of millimeters, and the microcapsules, with radius of hundreds microns and a shell thickness of hundreds of nanometers. In this section, the mechanical behavior of the self-healing material is studied using a multi-scale approach. It is assumed that the micro-capsules are evenly distributed in the polymer matrix. Therefore, the material is described using a representative volume element (RVE) that contains a single micro-capsule. A cubeshaped RVE is selected for this work, where a micro-capsule with a finite shell thickness is located in the center of the cube, as it can be seen in Fig. 15. The three fundamental dimensions of the system are the length of the cube $l$, the external radius of the micro-capsule $R_{e}$ and the shell thickness of the micro-capsule $t_{c}$. Using three-dimensional periodic boundary conditions (PBCs), the RVE represents an infinite system and can provide the mechanical response of an encapsulation-based selfhealing polymer. The periodic distribution of the micro-capsules is a strong hypothesis which does not take into account the randomness of the material. Yu et al. [62] showed that the particle clustering has effects in the failure mechanisms. A similar trend in particle reinforced metal matrix composites was found in ref. [63]. Further analysis of the randomness effects is out of the scope of this paper. 


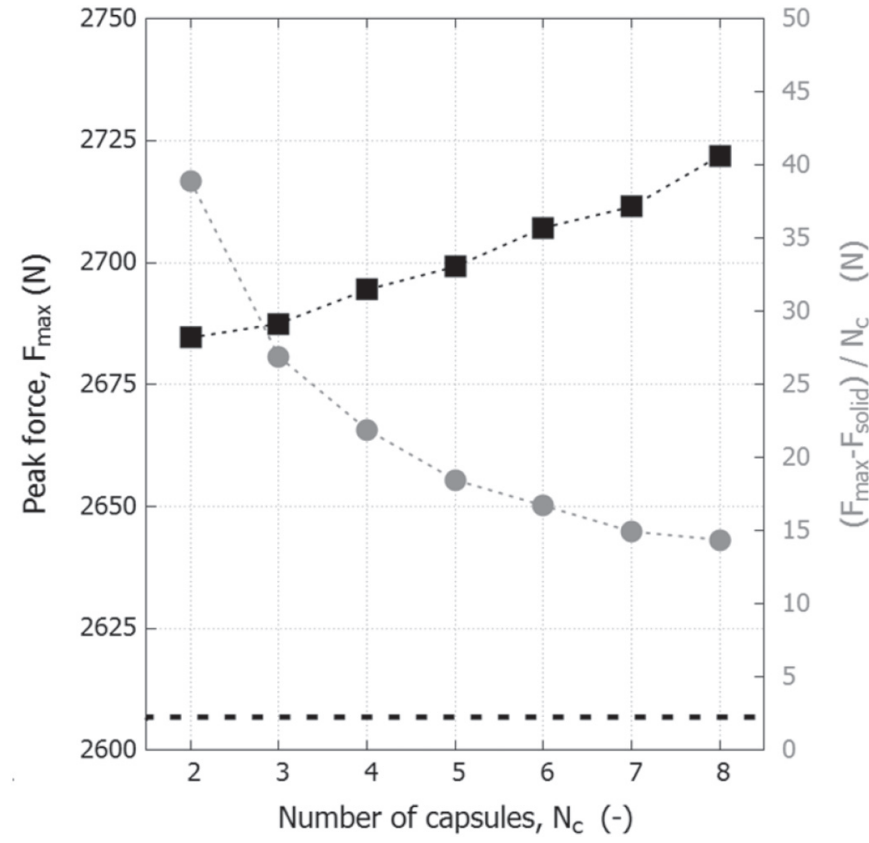

Fig. 13. Maximum force developed by beam with different number of capsules subjected to three-point-bending test.
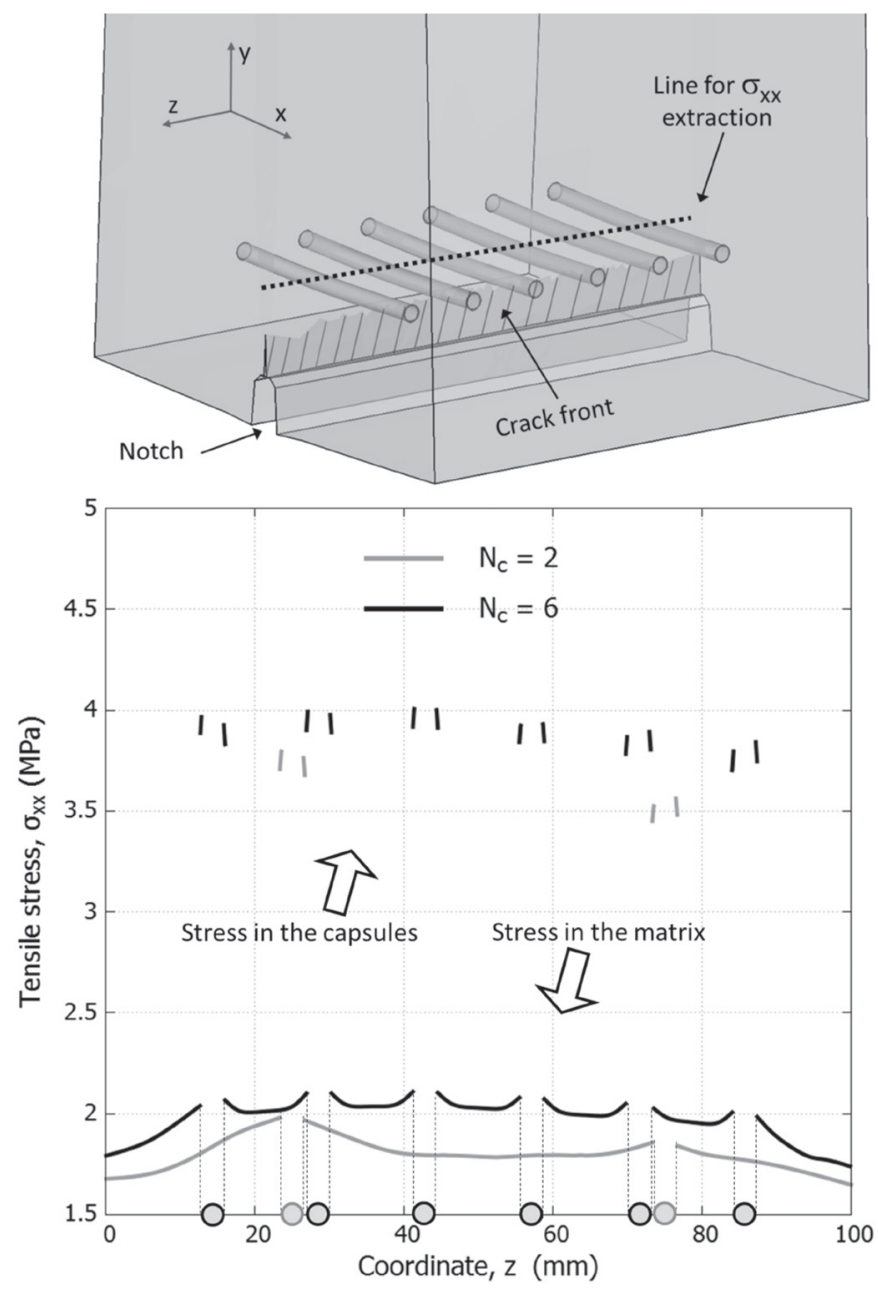

Fig. 14. Tensile stress profile $\left(\sigma_{x x}\right)$ passing through the beam depth. Two cases are compared: beam with 2 and 6 capsules.

\subsection{Representative volume element domain}

This micro-scale model can be described by the external radius $R_{e}$ and the volume fraction $V_{f}$ of the micro-capsule content, where $V_{f}$ is defined as the volume of micro-capsules divided by the total volume. Taking into account the periodicity, the RVE length $l$, which is the mean distance between micro-capsules, can be calculated from the volume fraction $V_{f}$ and $R_{e}$ as follows

$l=R_{e} \sqrt[3]{\frac{4 \pi}{3 V_{f}}}$.

The radius $R_{e}$ is an experimental value that ranges $25-250 \mu \mathrm{m}$, however, the volume fraction $V_{f}$ must be estimated. The range of $V_{f}$ can be calculated by assuming some hypotheses based on the expected healing behavior. These hypotheses are described as follows. The healing agent contained in a single micro-capsule must fill the crack plane around it. Assuming that the micro-capsules are periodically distributed, only one RVE with a horizontal crack in the equator is considered. It is also considered that the crack has a constant gap and the volume of healing agent spread in the crack gap is only the half of the micro-capsule volume. This last assumption takes into account that the micro-capsule has a shell thickness, the crack plane might have some roughness, and some of the healing agent might remain in the void after the capsule breakage.

This particular case does not include effects due to random distributions, different aspect ratio or orientation of the capsules [64], which are out of the scope of the present study.

Fig. 16 shows the minimum $R_{e}$ as a function of $V_{f}$ required to fill different crack gaps. In the light of this result, a volume fraction between 0.05 and 0.25 is necessary to fill a wide range of gaps between 5 and $50 \mu \mathrm{m}$ for the available micro-capsules, as it is marked by the gray area in Fig. 16. This range agrees with the experimental values [65-67].

\subsection{Numerical setup}

The present micro-scale model consists of two parts described by solid elements: the cubic matrix part with a spherical hole of radius $R_{e}$ and the spherical shell of the micro-capsule with thickness $t_{c}$ located in the hole. Each part has its own material properties and both are descried by extended finite elements. Similarly to the previous model from Section 3, quasi-brittle damage behavior is also used for the polymer matrix and micro-capsule. Likewise, there are cohesive surfaces between the external surface of the capsule shell and the internal surface of the matrix in order to account for the capsule-matrix interface (see Fig. 2).

PBCs have been implemented using node-to-node equation constraints. The global coordinate system is located in one of the bottom vertices of the RVE, with the axis along the edges of the RVE (see Fig. 15). The set of equations that describes the coupling between the displacements of one node and its counterpart on the opposite surface is given by

$u_{j}^{i+}-u_{j}^{i-}=\bar{\varepsilon}_{i j} \Delta i=u_{j}^{R P i} \quad i, j=x, y, z$

where $\bar{\varepsilon}_{i j}$ are the components of the average strain tensor, $\Delta i$ is the current length of the RVE along the direction $i$ and $u_{j}^{R P i}$ stands for the displacement component of the dummy node $R P i$. There are three dummy nodes in the model, one per direction, which are used to introduce the load condition. More detailed information about this implementation can be consulted in ref. [68].

The RVE is loaded under tension in Y direction via a quasi-static vertical displacement applied to the dummy node $R P Y$. Therefore, $u_{y}^{R P Y}=u_{y}^{\max } \tau / T$, where $\tau \in[0, T]$. This implementation makes it possible to take into account the Poison effect in the transverse plane $X Z$, in which case the displacements $u_{x}^{R P X}$ and $u_{z}^{R P Z}$ are free. All the shear components of the dummy nodes are set to zero because no shear deformations are expected taking into account the symmetry of the problem. The implicit solver of Abaqus/Standard has been used, where the total duration of the load has been set $T=1$ and the maximum 

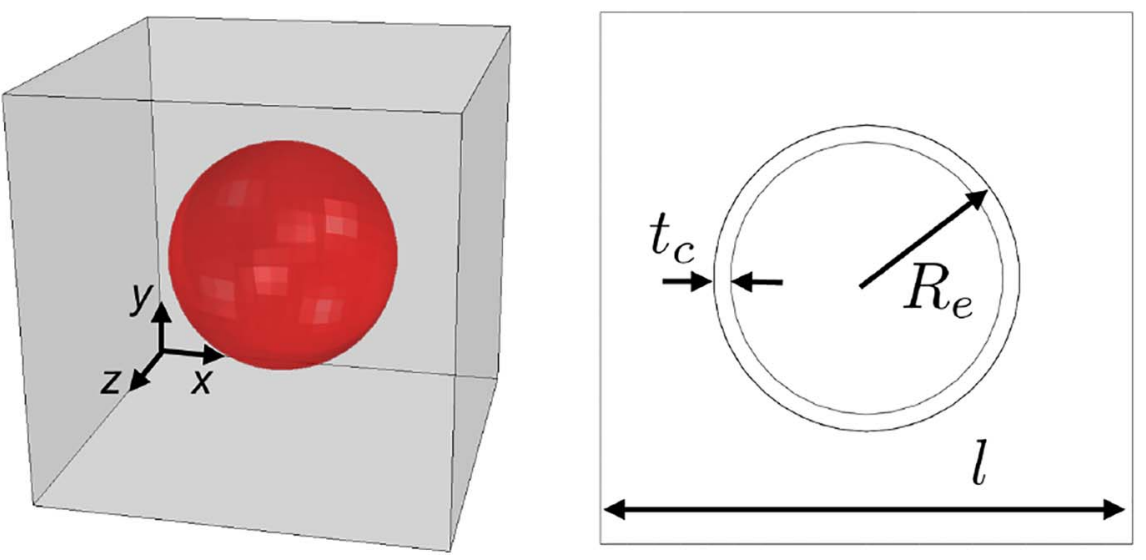

Fig. 15. Geometry of RVE with dimensions.

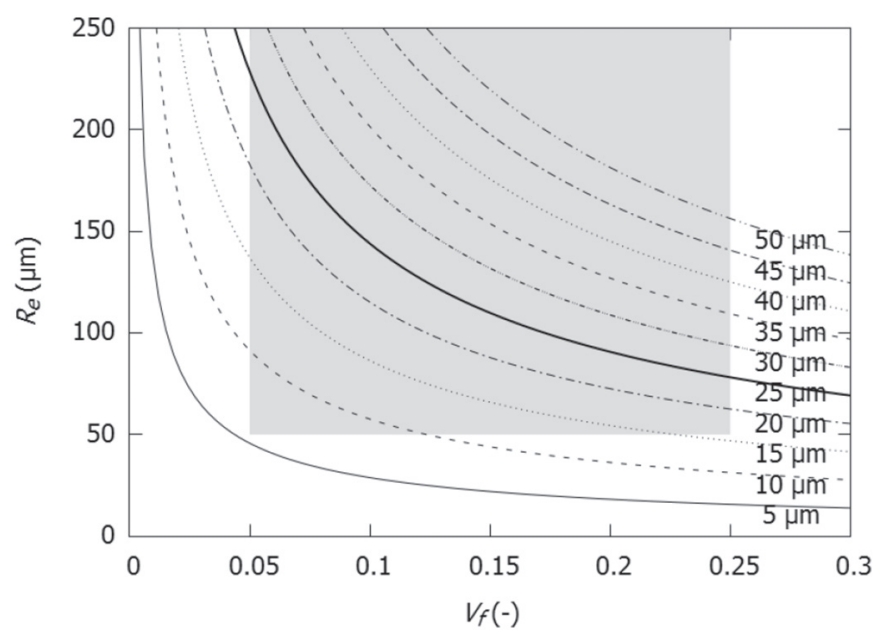

Fig. 16. Minimum capsule radius $\left(R_{e}\right)$ versus the capsule volume fraction required to fill different crack gaps (from 5 to $50 \mu \mathrm{m}$ ).

applied displacement was $u_{y}^{\max }=5 \mu \mathrm{m}$.

\subsection{Material properties}

Isotropic linear elastic material models have been used to describe the behavior of the polymer and the shell of the micro-capsules until failure. As mentioned, a quasi-brittle failure is assumed for each material based on the maximum strength and fracture energy (see Section 2.1). The typical materials used in an encapsulated-based selfhealing polymer consist in matrix made of epoxy resin (EPON) and a micro-capsule of urea-formaldehyde [65]. The mechanical properties of these materials are shown in Table 2.

It is important to mention that the epoxy properties for EPON are obtained from the standard macroscopic experiment with dimensions of millimeters. Therefore, these properties account for the bulk properties. Although the elastic properties do not significantly change when smaller samples are tested, the properties that describe the brittle

Table 2

Properties of self-polymer constituents.

\begin{tabular}{lllll}
\hline & $E(\mathrm{GPa})$ & $\nu(-)$ & $\sigma^{*}(\mathrm{MPa})$ & $G^{*}\left(\mathrm{~J} / \mathrm{m}^{2}\right)$ \\
\hline Matrix $\left(_{m}\right)[65]$ & 3.4 & 0.38 & 39 & 88 \\
Capsule $\left(_{c}\right)[69-71]$ & 3.7 & 0.33 & 55 & 100 \\
Interface $\left.{ }_{i}\right)$ & - & - & $1,2,5,10$ & $0.1,1,10$ \\
\hline
\end{tabular}

behavior can significantly differ at micro-scale due to the size effect [72]. Nevertheless, this study assumes that the epoxy properties are the same for all the studied RVE models.

On the other hand, the properties of the urea-formaldehyde shell are retrieved from micro experiment where micro-capsules are directly used [69]. The stiffness of the urea-formaldehyde $E_{c}$ has been calculated indirectly from a model of the compression test of a micro-capsule. Although the micro compression experiment is not dedicated to get the maximum strength $\sigma_{c}^{*}$, a range can be also extracted from the same experiments, 69-118 MPa. This range is over the typical value measured by different companies which make molded urea-formaldehyde, $55 \mathrm{MPa}$. In this study, we have assumed that $\sigma_{c}^{*}=55 \mathrm{MPa}$ to perform the simulations. Finally, the fracture energy $G_{c}^{*}$ is estimated from the adhesive experiments performed with urea-formaldehyde [71]. We have assumed a value of $100 \mathrm{~J} / \mathrm{m}^{2}$ which matches with the typical fracture toughness of other thermosets measured in standard experiments.

After the synthesis of the micro-capsules with the healing agent encapsulated [73], they are mixed with the resin and cured under standard conditions. A good affinity between the shell and the epoxy is expected. Indeed, the experimental result of a crack which propagates through the self-healing material shows that the interface is usually strong enough to break the capsules. In a few cases, there are debonds of the micro-capsules from the epoxy [74]. To the best of the author's knowledge, there are no direct experimental values for the properties of the interface. Therefore, a range of values is investigated as numerical parameter, see Table 2.

\subsection{Mesh convergence}

The mesh used in this micro-scale model is constructed with 8-node linear brick elements with full integration (C3D8 in Abaqus nomenclature [22]). After a study of mesh convergence similar to that performed for the concrete beam model (see Section 3.4), the average element size for all RVEs with different $V_{f}$ was fixed to $15 \mu \mathrm{m}$. The aspect of the resultant mesh is showed in Fig. 17. Taking into account the interface properties, this size is below the value of the cohesive zone length size discussed in [53]. Regarding the micro-capsule, two elements through the thickness of the shell have been selected. It has been checked that using more elements through the thickness does not increase the accuracy, although increases noticeably the computational effort.

\subsection{Crack evolution with and without precrack}

Due to the geometry of the RVE and the imposed PBCs, one single horizontal crack located in the middle of the RVE is expected at the end of the simulations. Two different scenarios are studied until complete failure of the RVE (see Fig. 18). In the first scenario without initial precrack, the initiation criteria of maximum principal stress will 


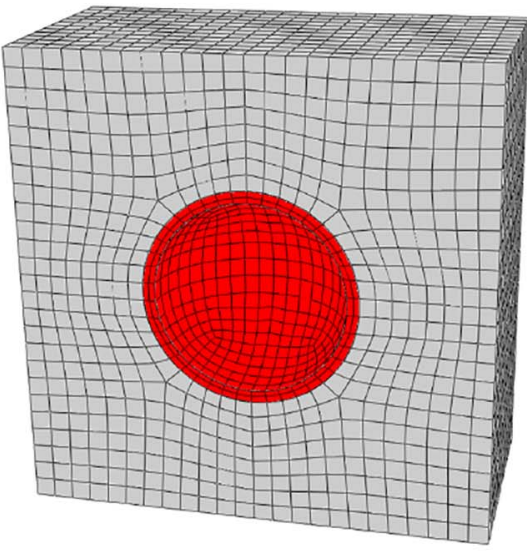

$v_{f}=0.05$

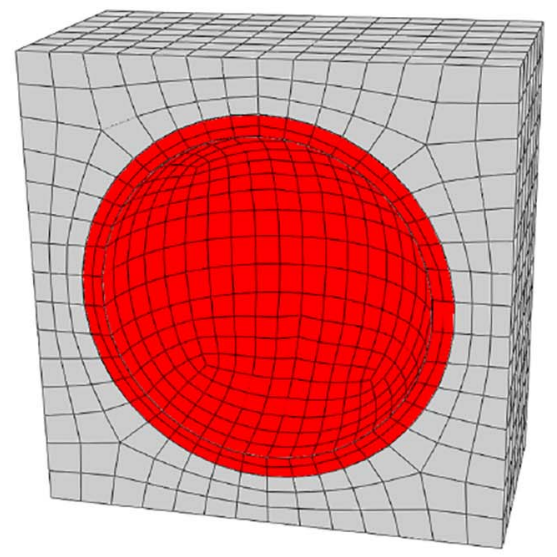

$$
v_{f}=0.25
$$

Fig. 17. Geometry of RVE with dimensions.
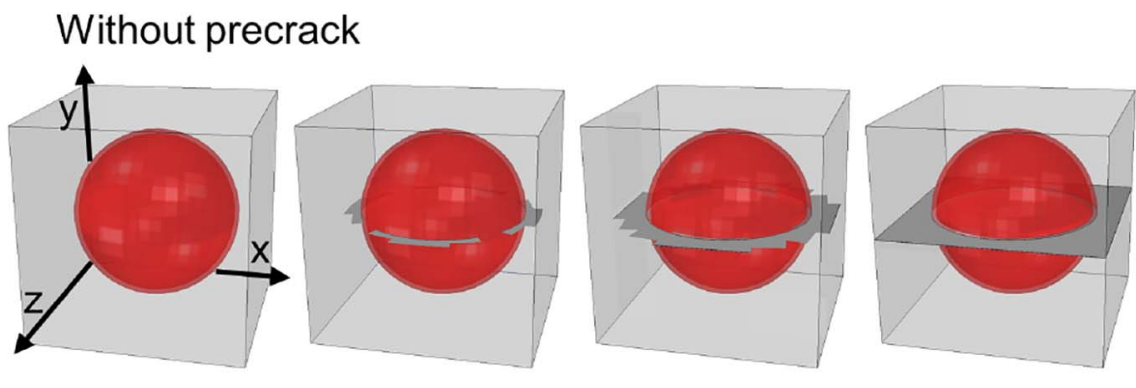

\section{With precrack}
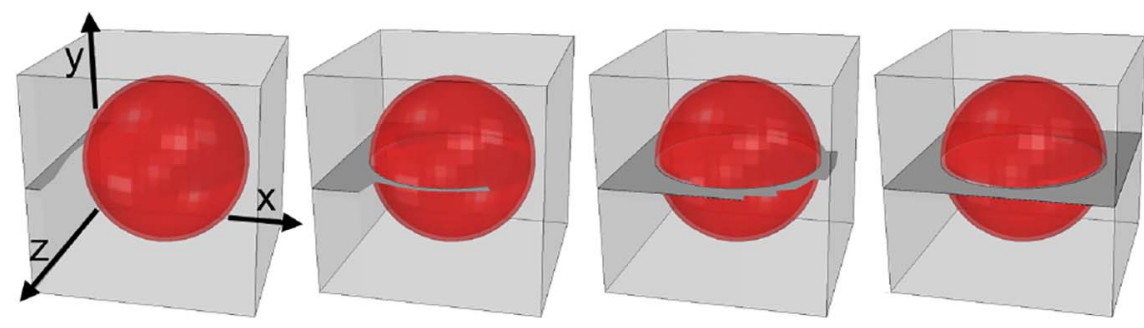

Fig. 18. Crack evolution without and with precrack.

introduce horizontal cracks on the equator of the micro-capsule. Because the matrix has lower maximum strength, the cracks are located first in the matrix material. Then, the cracks will grow through the matrix and the shell until they generate a complete horizontal free plane. In the second scenario, a precrack is introduced horizontally close to one of the lateral surfaces at middle height of the RVE (see left image of "with precrack" sequence from Fig. 18). This precrack, located at $(x=0, y=l / 2)$ and along the $z$ direction, cuts the first row of elements in the middle (the reason of this requirement will be explained in Section 5.1). In this scenario, the PBC equations that couple the $y$ displacements between nodes from opposite surfaces in $x$ direction are eliminated. This modification of the PBC boundary conditions avoids the restriction imposed by the vertical periodicity and makes possible the crack propagation along the $x$ direction. Under the imposed tension load, the crack will propagate horizontally through the matrix until reaching the shell of the micro-capsule. Then, the crack will embrace the shell through the equator. Finally, once the capsule is fully surrounded, the crack continues through the matrix and the shell until the complete division of the whole domain in two parts (see right image of "with precrack" sequence from Fig. 18).

It is important to mention, that in both scenarios the default implementation of Abaqus XFEM has been used. Therefore, new cracks cannot be initiated in an enriched region with a crack under propaga- tion. This limitation affects the cracking sequence in the scenario "with precrack" as it will be discussed in Section 5.2. Nevertheless, the homogenized stress-strain behavior of the RVE is not affected.

In the next section, the crack initiation and propagation through the matrix and the shell of the micro-capsule have been numerically studied for both scenarios as function of the geometrical parameters, (volume fraction $V_{f}$ and the geometric ratio $t_{c} / R_{e}$ ) as well as the interface properties.

\subsection{Breakage and debonding of micro-capsules}

In order to have a fully functional micro-encapsulation-based selfhealing polymer, it is essential that micro-capsules break when the crack reaches them (see left state in Fig. 19). In case of micro-capsule debonding, the crack simply grows through the epoxy matrix and the interface. As a consequence, the micro-capsules detach from the matrix without cracking themselves, as it is represented in the right image of Fig. 19.

The sequence of micro-capsule debonding can be described generally for both scenarios: "without precrack" and "with precrack". Under the imposed vertical load, the debonding starts at the area with maximum shear, which is located at $\pm 45^{\circ}$ with respect to the horizontal plane of the micro-capsule equator. This feature has 

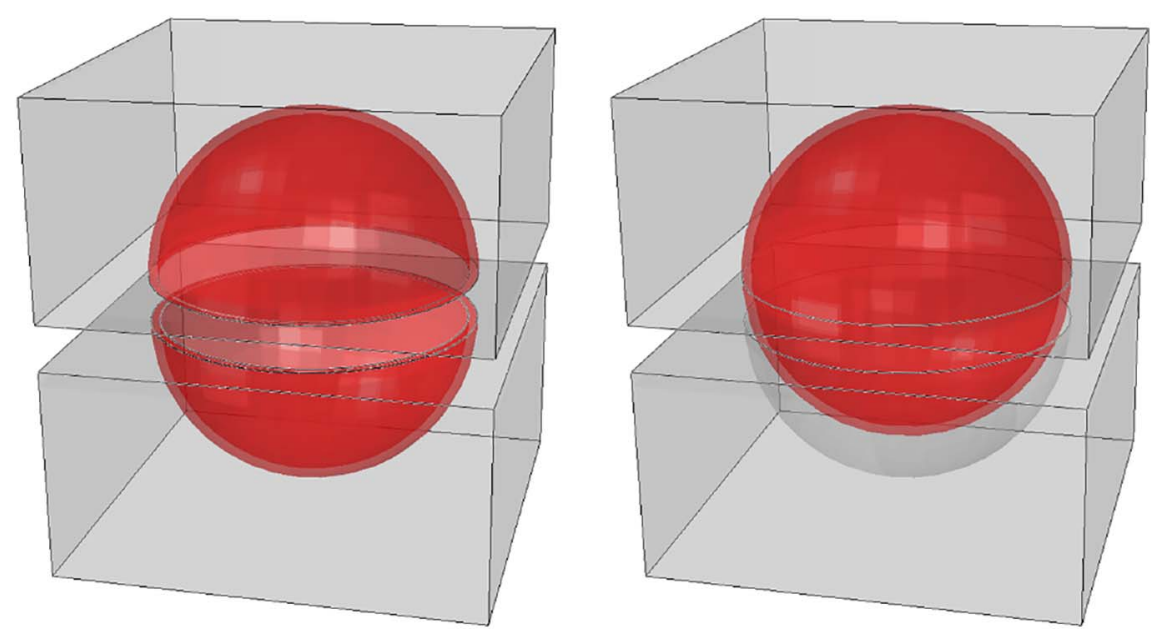

Fig. 19. Break (left) and debond (right) of the micro-capsules after the crack propagation along the horizontal middle plane.

similarly been observed by using cylindrical capsule under far-field tensile stress [75]. Before the initialization or propagation of any crack in the matrix, the debonding area growths around the initial debonding at $\pm 45^{\circ}$, leaving attached the top and bottom part of the interface. Then, when the matrix crack propagates through the horizontal plane, the interfacial debonding is completed and the micro-capsule is fully detached from one of the spherical caps, top or bottom. Rounding errors during the simulation leads to this non-symmetric debonding despite the inherent symmetry present in the model.

The breakage or debonding of the micro-capsule depends on the coupled effect of the material properties and the geometrical parameters. In this section, the limit conditions to produce the breakage or debonding of the capsule are studied within a range of $\sigma_{i}^{*}$ for different $t_{c} / R_{e}$. According to Table 2, four interface maximum tensile strengths $\left(\sigma_{i}^{*}=1,2,5,10 \mathrm{MPa}\right)$ and three values of the interface fracture energy $\left(G_{i}^{*}=0.1,1,10 \mathrm{~J} / \mathrm{m}^{2}\right)$ have been used. The simulations have been carried out using four different geometric ratios, namely, $t_{c} / R_{e}=0.011$, $0.022,0.055,0.111$. The geometric ratios correspond to micro-capsules with four different thicknesses, $t_{c}=1,2,5,10 \mu \mathrm{m}$ and constant external radius $R_{e}=90 \mu \mathrm{m}$. In all cases, two bounding values of volume fraction from Fig. 16 have been analyzed $\left(V_{f}=0.05,0.25\right)$. Finally, the scenarios without and with precrack have also been included in the present study.

The different fracture responses have been analyzed and compared using the homogenized true stress-strain curves. The homogenized true stress is calculated from the true stress computed in each element and taking into account the whole volume of the RVE (including the void). The homogenized true strain is directly obtained from the applied vertical displacement set in the PBC. Fig. 20 shows the response of the homogenized true stress as a function of the homogenized true strain for three geometric ratios, $t_{c} / R_{e}=0.022,0.055,0.111$ and volume fraction $V_{f}=0.25$. The interface strength values $\sigma_{i}^{*}$ are $1,2,5$ and 10 $\mathrm{MPa}$, while $G_{i}^{*}$ has been kept constant and equal to $1 \mathrm{~J} / \mathrm{m}^{2}$. For each geometric ratio $t_{c} / R_{e}$, the scenarios without and with precrack have been studied. As reference, every plot in Fig. 20 also depicts the homogenized mechanical response generated by a RVE with a void of radius $R_{e}$, i.e., the void case. The lower value of $\sigma_{i}^{*}$ for each subfigure represents the debonding limit (solid lines), which means that the micro-capsule debonds during the crack propagation through the matrix for $\sigma_{i}^{*}$ equal or below this value. On the other hand, the upper value of $\sigma_{i}^{*}$ represents the limit of capsule breakage (dashed lines). This limit indicates that the micro-capsule will break if the used value of $\sigma_{i}^{*}$ is equal or larger than it. From the stress-strain curves, the homogenized Young's modulus (or stiffness) $E$ can be obtained from the initial slope. Similarly, the maximum tensile strength, denoted by $\sigma_{\max }$, is obtained from the peak value of the curve. As Fig. 20 shows, debonding or rupture of the capsule cannot be distinguished when the geometric ratio is small $\left(t_{c} / R_{e}=0.022\right)$. This difference becomes more visible as this ratio increases (see cases $t_{c} / R_{e}=0.055$ and $t_{c}$ /
$R_{e}=0.111$ )

As expected, after full breakage (or debonding), the response obtained from all cases converges to the stress-strain response given by the void case. It has been found that these limit values of breakage (or debonding) of the capsule are the same regardless the presence of the precrack. The main difference between both scenarios comes from the fact that $\sigma_{\max }$ reaches lower values in the scenario with precrack for all the cases: the total area of crack propagation is reduced when the precrack is introduced.

On the other hand, as a healthy exercise to check the reliability of homogenization process, a simple calculation has been done. In the case of a RVE with a void, it has been checked that the total fracture energy obtained from the integral of the curve given by the homogenized stress versus the imposed displacement (i.e., the area below the curve) retrieves exactly the value of fracture energy of the pure matrix $\left(G_{m}^{*}\right)$ multiplied by the area of the horizontal crack. In that sense, the presence of the micro-capsule increases the total fracture energy, more noticeably for the case of breakage, which leads to a visible increment of $\sigma_{\max }$.

The maximum tensile strength $\sigma_{\max }$ for each $t_{c} / R_{e}$ at different $\sigma_{i}^{*}$ are plotted in Fig. 21. The results of the histogram bars correspond with the scenario without precrack, while the results with circles correspond with the scenario with precrack. The cases with micro-capsule breakage are labeled as "B" and the cases with capsule debonding are labeled as "D". For the lowest geometrical ratio $t_{c} / R_{e}=0.011$, the micro-capsule breaks at the smallest $\sigma_{i}^{*}$ studied, therefore, no debonding is possible when $\sigma_{i}^{*}$ is increased. In addition, using this geometric ratio, $\sigma_{\max }$ does not change for any value of $\sigma_{i}^{*}$, and whose value is slightly higher than the value provided by the void case. When $t_{c} / R_{e}$ increases, the debonding limit increases as well. Then, it is necessary to ensure a stronger interface to avoid capsule debonding in the self-healing polymer. If $\sigma_{i}^{*}$ is equal or larger than $10 \mathrm{MPa}$, the micro-capsules break for the studied range of $t_{c} / R_{e}$, and $\sigma_{\max }$ increases when $t_{c} / R_{e}$ increases as well. On the contrary, a weak interface with low $\sigma_{i}^{*}$ cannot guarantee the breakage of the micro-capsules, and therefore, the expected self-healing behavior might not be obtained.

The same simulations have been performed for the smallest volume fraction considered in this work, $V_{f}=0.05$. For the selected range of $t_{c} /$ $R_{e}$ and $\sigma_{i}^{*}$, the results are the same to the case $V_{f}=0.25$ (Fig. 21). Furthermore, simulations with different interface fracture energy, $G_{i}^{*}=0.1,1,10 \mathrm{~J} / \mathrm{m}^{2}$, have been done with $t_{c} / R_{e}=0.055$ and $V_{f}=0.25$. When $\sigma_{i}^{*}$ is equal or larger than the breakage limit of 5 $\mathrm{MPa}$, no effect is observed due to the fact that the crack cannot propagate through the interface. When $\sigma_{i}^{*}$ is equal or smaller than the debonding limit of $2 \mathrm{MPa}$, the stress-strain response changes slightly compared to the results of $G_{i}^{*}=1 \mathrm{~J} / \mathrm{m}^{2}$. As summary, for $G_{i}^{*}=10 \mathrm{~J} / \mathrm{m}^{2}$, $\sigma_{\max }$ is equal to the reference case $(20.4 \mathrm{MPa})$, whereas for $G_{i}^{*}=0.1 \mathrm{~J} /$ $\mathrm{m}^{2}, \sigma_{\max }$ drops down to the value of the void case (19.7 MPa).

Despite the typical geometric ratio of real micro-capsules $\left(t_{c}\right)$ 

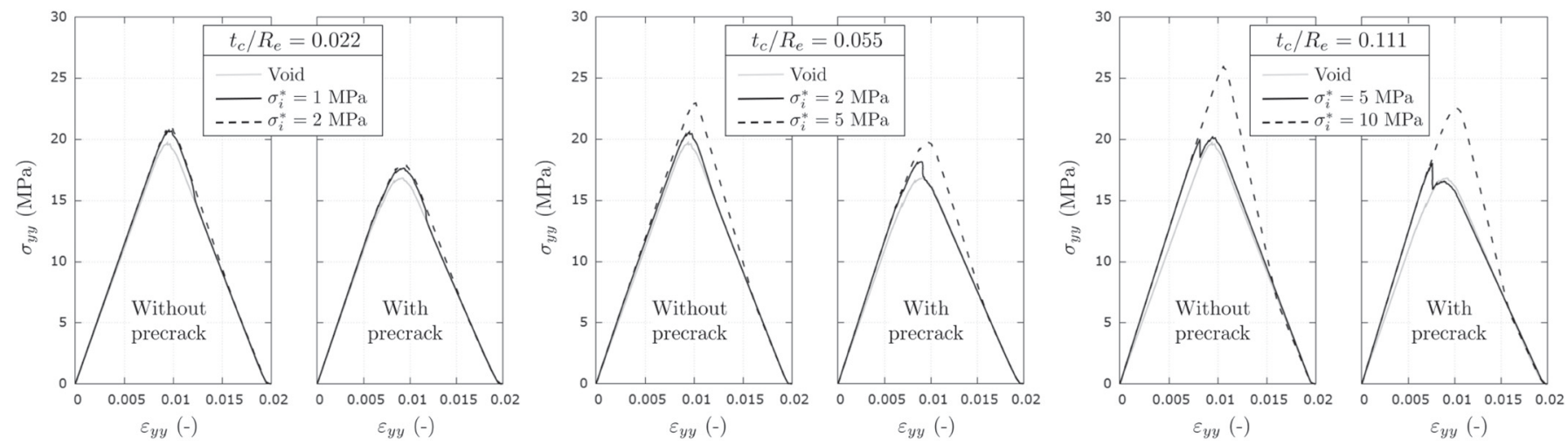

Fig. 20. Stress-strain curves for different geometric ratios $\left(t_{c} / R_{e}=0.022,0.055,0.111\right)$ and interface strength $\left(\sigma_{i}^{*}\right)$. Capsule volume fraction $V_{f}=0.25$.

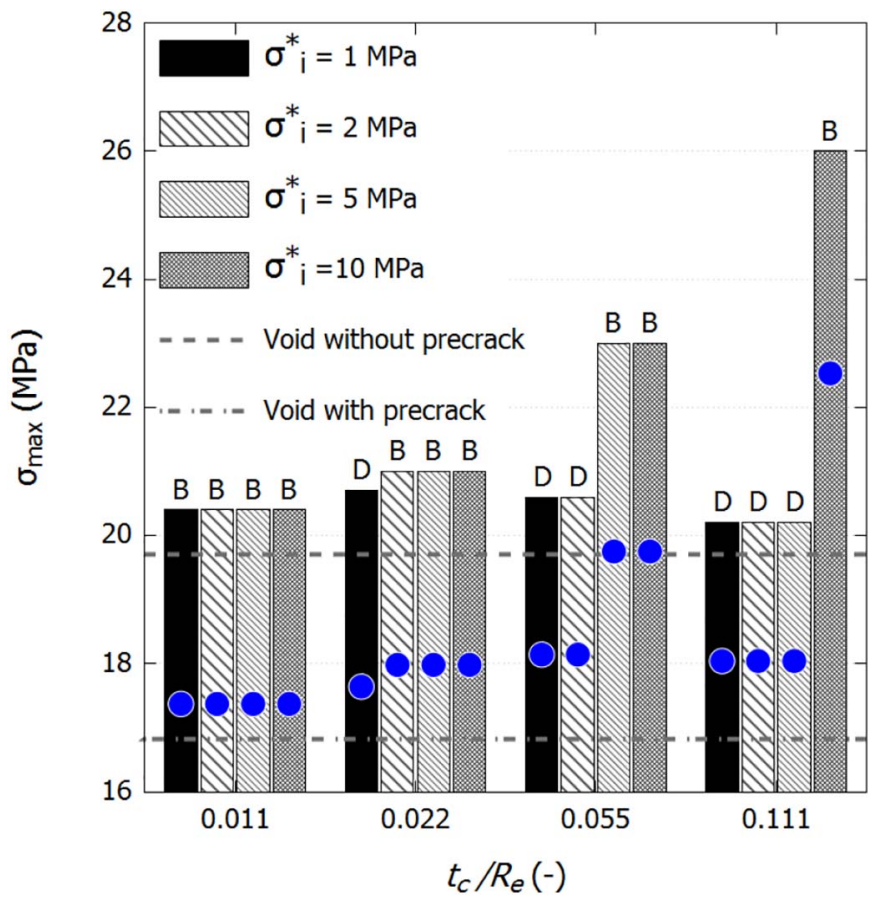

Fig. 21. Maximum stress until failure of the RVE as a function of the geometric ratio and different interface strength levels (bars: without precrack, circles: with precrack).

$R_{e}=0.004$, see ref. [65]) is still below the lowest value used in this work $\left(t_{c} / R_{e}=0.011\right)$, no big differences should be expected from the homogenized mechanical point of view. The stress-strain behavior of the geometric ratio $t_{c} / R_{e}=0.011$ behaves almost like the void case (this is the reason why this case is not shown in Fig. 21). Therefore, it is expected that lower ratios than 0.011 will exhibit the same stress-strain behavior as the void $\left(t_{c} / R_{e}=0\right)$, and then, with a maximum strength much closer to the void case. As a consequence, if $t_{c} / R_{e}<0.011$, it is expected that the capsule will always break regardless the value of $\sigma_{i}^{*}$ within the interval studied in this paper.

In that sense, from the FEM point of view, the numerical analysis of much thinner shells would require the usage of shells elements. In this case, the same combined strategy of XFEM and cohesive surfaces is less obvious and it would demand considerable additional research.

It is worth mentioning that a strong interface between microcapsules and the matrix is usually expected. Experimental results showed that the majority of micro-capsules broke along the crack path, and only a very little fraction of them debonded from the matrix [74]. Therefore, in the following section, the mechanical properties of a homogenized encapsulation-based self-healing polymer are studied as function of the geometric parameters assuming a strong interface $\left(\sigma_{i}^{*}=10 \mathrm{MPa}\right)$.

\subsection{Mechanical properties prediction}

It is known that the mechanical behavior of the polymer change with the amount of micro-capsules $[65,67]$. As a general behavior, the stiffness of the material and the maximum strength decrease when the $V_{f}$ of micro-capsules increases. In this section, simulations without initial precrack are used to calculate the effective response of the Young's modulus and the maximum tensile strength. The effective Young's modulus $E$ and maximum tensile strength $\sigma_{\max }$ are obtained from the homogenized strain-stress curves. Both effective values have been calculated under the hypothesis of PBC described before, therefore some difference with the real ones are obviously expected. Nevertheless, the effective values obtained in these simulations are useful to follow up the trend of these properties as function of $V_{f}$ and $t_{c} / R_{e}$.

Fig. 22 shows the effective stiffness and maximum strength as function of the volume fraction $V_{f}$ for several cases with different geometrical radius $t_{c} / R_{e}$. The stiffness $E$ decreases with the $V_{f}$ as expected, because the inclusion of micro-capsules can be considered like the inclusion of voids in the matrix. When the geometrical radius $t_{c} / R_{e}$ decreases, the decrease of $E$ is more pronounced until reaching the limit at $t_{c} / R_{e}=0$, which is the case of the void.

Although the stiffness $E$ obtained from the experiment also decreases with $V_{f}$, its value is higher than the values from the simulations (see experimental data by Brown in Fig. 22). One reason to explain this difference might be that the core inside the micro-capsule (self-healing agent with some water or air) can contribute to the homogenized stiffness. Another plausible reason might be that the properties of the epoxy matrix change when it is cured with the embedded microcapsules. These features lay out of the scope of the present paper.

The homogenized maximum strength $\sigma_{\max }$ also decreases when $V_{f}$ increases, as it is clearly shown in Fig. 22. Indeed, $\sigma_{\max }$ decreases abruptly when $V_{f}$ equals $5 \%$, and then decreasing softly after such value. It must be noted that, although with different values, this tendency is also clearly observed in the experimental works from other authors $[65,67]$.

It is important to recall that this model uses PBC. The periodicity produces correctly the interaction of stresses between capsules, however it also introduces the limitation of having a perfect distribution of micro-capsules. In a random distribution of micro-capsules, a combination of normal and shear stress are expected around the micro-capsules, what would lead to different $\sigma_{\max }$ and different location of the crack initiation. Moreover, a periodic distribution assumes a prefixed crack density because all the capsules will break at the same time. This is a strong hypothesis and it might deviate the numerical value from the experimental ones (where possibly only one crack is running through the whole material). Although using PBC involves some limitations, the homogenized material properties predicted by using the micro-model show a good agreement with the experimental trend. This model can be very useful to improve and to understand new encapsulation-based selfhealing polymers. 


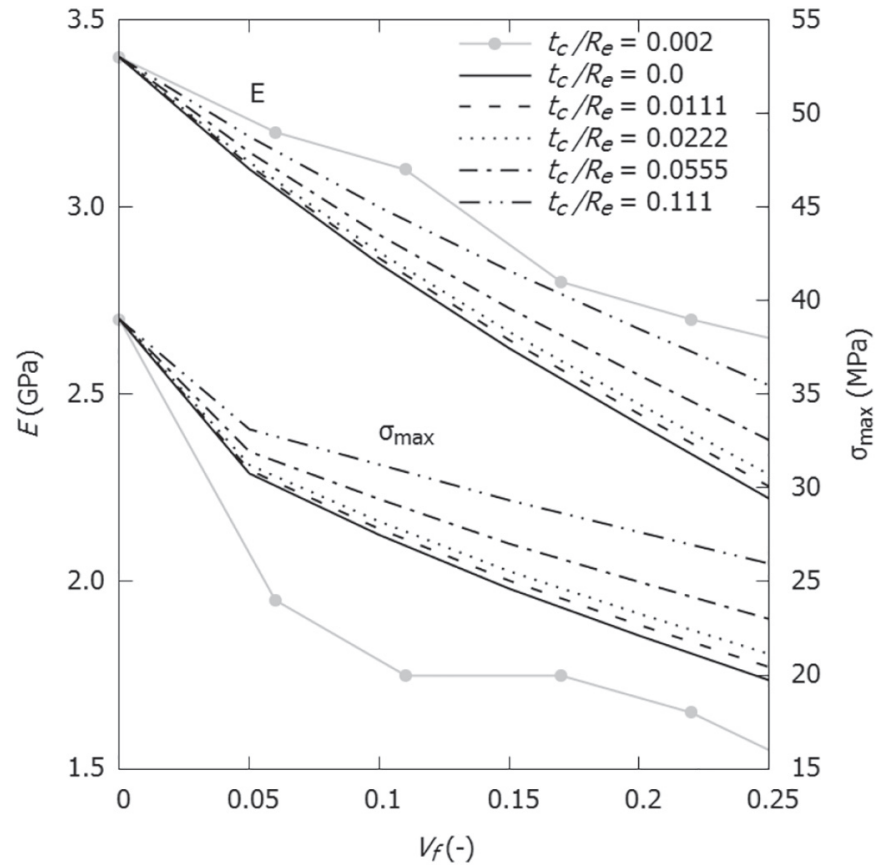

Fig. 22. RVE homogenized stiffness $E$ and maximum tensile strength $\sigma_{\max }$ as function of the volume fraction $V_{f}$ and the geometrical ratio $t_{c} / R_{e}$. (Circles: experimental results by Brown et. al [65]).

\section{Modeling limitations of the implementation}

This section discusses some important features that any researcher should be aware of in case of using the current commercial implementation, which might strongly affect the reliability of the crack paths. Although this finite element package makes it possible to simulate arbitrary crack propagation, it is however very important to know and assess the limitations of the implementation used to produce realistic and reliable results.

Here two limitations are discussed. The first limitation is related with the progression of the crack path when it grows close to the element boundary. The second limitation is related with the default implementation used to manage the initiation of multiple cracks in the same enriched region where a crack already propagates.

\subsection{Crack path close to the element boundary}

A simple model has been created to establish the simulation response when a crack path is spreading alongside and very close to the boundary between two elements. For the sake of simplicity, this model has been created assuming plane strain conditions, although the same behavior and anomalous response is obtained under plane stress and three-dimensional conditions.

Fig. 23 (left) shows the geometry of the specimen used in this benchmark. It consists in a rectangular plate with dimensions $W=10$ $\mathrm{mm}, H=20 \mathrm{~mm}$ and depth of $1 \mathrm{~mm}$. In order to get the reaction forces, two reference points are defined (RPT, RPB). These points are linked to the node lines (NLT, NLB) through a constraint given by

$u_{y}^{\mathrm{RPp}}-u_{y}^{\mathrm{NLp}}=0$,

where $u_{y}$ is the vertical displacement and $p=T, B$. The specimen is stretched using a displacement-type controlled procedure. This action has been done applying a prescribed displacement magnitude of $0.005 \mathrm{~mm}$ in vertical degree of freedom to the reference points. To avoid rigid body movements along the $\mathrm{x}$-axis, the upper and lower right corner nodes are horizontally blocked. Regarding the mesh, a 4-node bilinear plane strain quadrilateral elements (CPE4R for reduced integration and CPE4 for full integration) were used for this example. The size of the elements, $l_{e}$, was set $0.357 \mathrm{~mm}$ and the total number of elements generated was 1568 . The elastic properties of the plate are those for the matrix concrete showed in Table 1 . The values of the fracture parameters were modified in order to increase slightly the degree of brittleness of the matrix. The selected parameters were $\sigma_{m}^{*}=3.7 \mathrm{MPa}$ and $G_{m}^{*}=12 \mathrm{~J} / \mathrm{m}^{2}$.

In order to simplify the problem, this model does not account for crack initiation, and therefore an initial precrack has been included (see white segment in Fig. 23). This precrack consists in a small segment of $2 \mathrm{~mm}$ length placed on the left side of the central region of the plate. To assess the anomalies during the crack propagation process, the parameter $\Delta$ is used to set the distance between the precrack and a boundary element, as it is shown in Fig. 23 (right). Four scenarios have been analyzed, namely:

- The precrack lays exactly in the middle of the element, i.e., $\Delta /$ $l_{e}=0.5$,

- The precrack is above the element boundary with $\Delta / l_{e}=0.028$,

- The precrack is above the element boundary with $\Delta / l_{e}=0.0028$ and

- There is a full overlap between the precrack and element boundary, i.e., $\Delta / l_{e}=0$.

Fig. 24 shows the mechanical response of the precracked solid plate under tension and Fig. 25 shows the trajectory of the crack in two stages: when the peak force occurs (Fig. 25a) and when the maximum displacement has been reached (Fig. 25b).

This figure clearly exhibits the sensitivity of the crack trajectory as a function of the distance to the element boundaries. Except for the case where the crack line overlaps exactly with the boundary between elements, the mechanical response is very similar, as it can be appreciated in Fig. 24. However, there are still some differences in the crack pattern, as for instance, the small artificial deviation when the initial separation crack-boundary is $\Delta / l_{e}=0.0028$. On the other hand, a very anomalous behavior is found when the crack overlaps with the boundary. As it is shown in Fig. 25b, some nodes are not able to get a full separation. This artificial clamping promotes an extremely high level of stress in those elements which are surrounding the crack. Moreover, quite visible hour-glassing in the elements that surround the crack lips is generated. Because of these large stresses, a sudden multicracking event is achieved when all cracked elements reach the crack initiation condition, despite the fact that there is no full physical separation yet. The implication of this anomalous behavior is shown in the force-displacement curve from Fig. 24 (case $\Delta / l_{e}=0$ ): an artificial increase of the fracture toughness is produced. This case has been carried out using reduced integration, however similar results using full integration were also achieved.

\subsection{Multiple cracks in an enriched region}

It is worth mentioning that the Abaqus/XFEM implementation cannot treat the initiation of cracks as a fully independent event. Abaqus makes use of the internal continuous damage variable STATUSXFEM, whose values are within the interval $[0,1]$. A value equal to 0 indicates a fully intact element. A situation with $0<$ STATUSXFEM $<1$ indicates a certain level of damage that corresponds to the softening region of Fig. 1. Within this region, although the solid element is already damaged, it can still carry stresses. If STATUSXFEM equals 1 , the element is fully damaged and stresses cannot be transferred anymore. The behavior by default does not allow for initiating a new crack in the same enriched region until the current running crack is fully broken (i.e., this running crack must fulfill exactly STATUSXFEM $=1$ in all the damaged elements). However, it might occur that some matrix elements in the same enriched domain already satisfy the crack initiation criterion even when the elements containing the tip of the existing crack still meet the condition STATUSXFEM $<1$. This situation represents a drawback, and as a consequence, it might lead to doubtful results. Nevertheless, this limitation can be overcome by using the undocumented option *enrichment activation, activate=multicracks within the section ${ }^{*} \operatorname{STEP}$ in the Abaqus input file. 


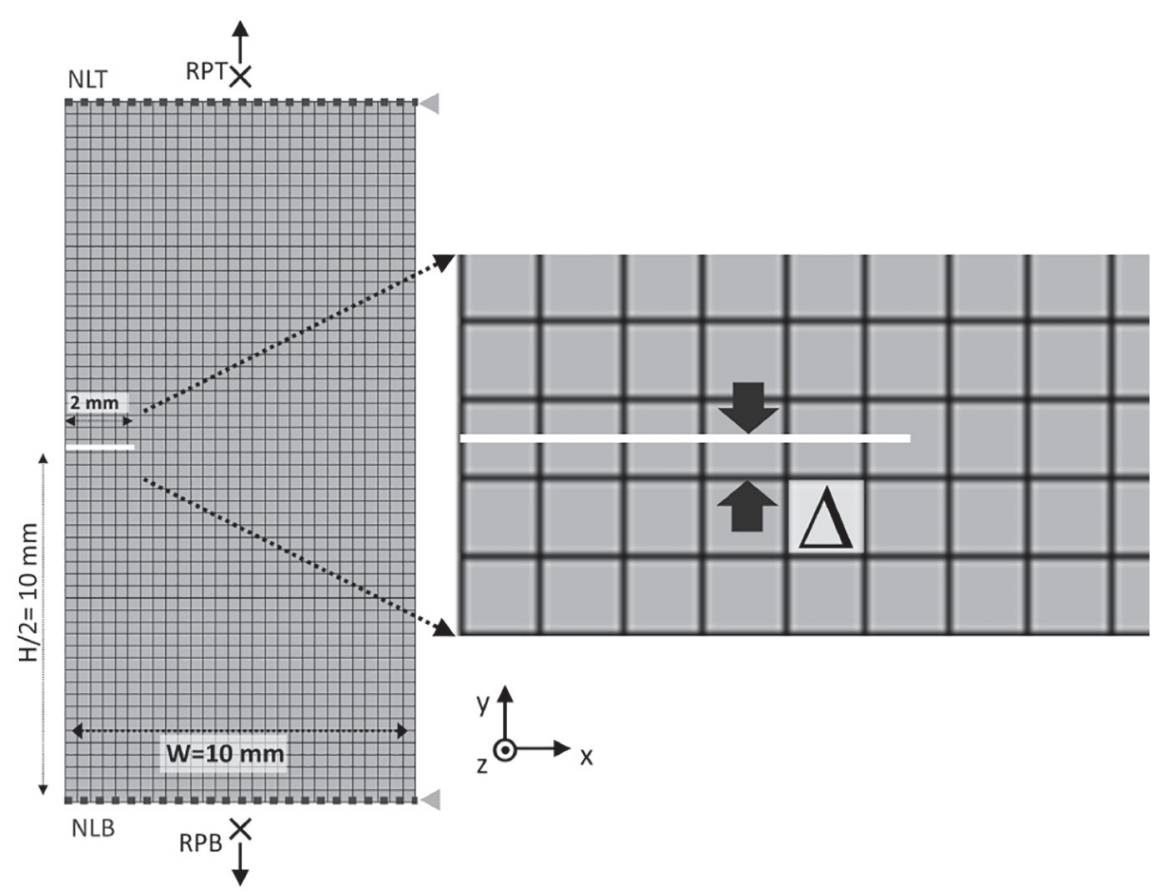

Fig. 23. (Left) Precracked solid plate specimen. (Right) Detail of the gap between the precrack and an element boundary.

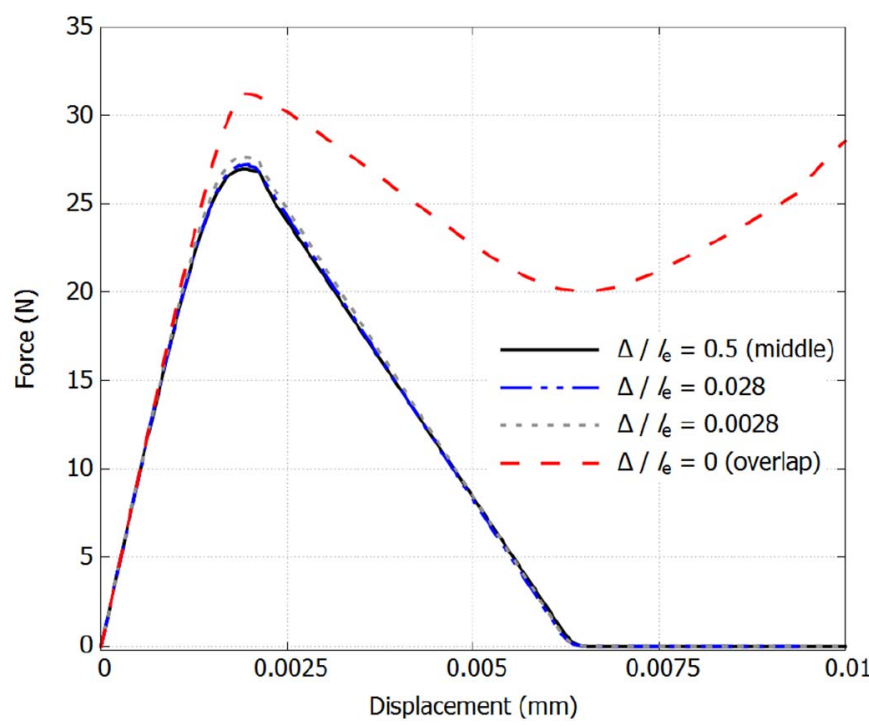

Fig. 24. Reaction force versus displacement curve obtained from precracked solid plate specimen.

The activation of multicrack mode has been tested for both scenarios described in Section 4.6: without and with precrack. In the first scenario, without precrack, multiple initial cracks are generated simultaneously around the equator, see without precrack images in Fig. 26. This initial multicracking is allowed by the default setup whenever it is produced exactly within the same time increment. Therefore, regardless the activation of the option multicracks, the same results are obtained as in Section 4.6. Hence, the propagation and coalescence of these initially-created multiple cracks in a single big horizontal crack seems to be a realistic result, where the elements in this horizontal region undergo the highest stress.

In the second scenario, with precrack, no secondary cracks can be developed by using the default setup (multicracks is not activated). Therefore, only the propagation of the prescribed precrack is produced (see "with precrack" images in Fig. 18). However, it has been checked that the stresses of the matrix elements around the micro-capsule equator already fulfill the initiation criterion when the crack begins to touch the capsule shell. Therefore, the simulation by activating multicracks shows a different cracking sequence in the matrix, see "with precrack" images in Fig. 26. First, the precrack propagates through the matrix until it reaches the shell, as usual. But this time, secondary cracks in the matrix elements initiate diametrically opposed to the crack front. Hence, both crack fronts propagate around the capsule equator to coalesce in a single crack. Finally, this single crack ends its propagation through the entire matrix and capsule that splits up the cubic domain in two halves. Unfortunately, the solver presents difficulties to get convergence in the last time-step because an unrealistic number of cracks are suddenly initiated. This effect leads to finish the simulations abruptly. Nevertheless, albeit the fracture sequence is different due to the presence of secondary cracks, it must be highlighted that the homogenized stress-strain response of the RVE does not essentially change compared to the case when the feature multicracks is not activated. Therefore, for sake of simplicity, the default setup implementation has been used in the current study in order to avoid convergence problems at the end of the simulations.

\section{Conclusions}

This paper presents two 3D models based on the combination of the eXtended Finite Element Method (XFEM) and the Cohesive Surfaces technique (CS) to study the process of fracture in encapsulated selfhealing materials. A complete summary of both numerical approaches is presented, as well as their main features, limitations and similarities between them. The interpretation of the material properties required for each approach has also been discussed and correlated to the experimental measurements available from the practical point of view. Using this methodology, the role of the bonding strength between the capsule and the matrix has been studied in two different material systems: concrete and polymers. The main purpose of this paper is to show how this methodology can be useful for experimentalists in order to get a better understanding of the interaction between cracks and capsules.

A realistic-size concrete beam model under three-point bending load has been developed. This setup is used to assess the encapsulationbased self-healing strategy in concrete structures at lab level. A macroscale model has been used to investigate the effect of the insertion of tubular glass capsules in the overall bending response. In order to make the computation affordable and usable for experimentalists in the 

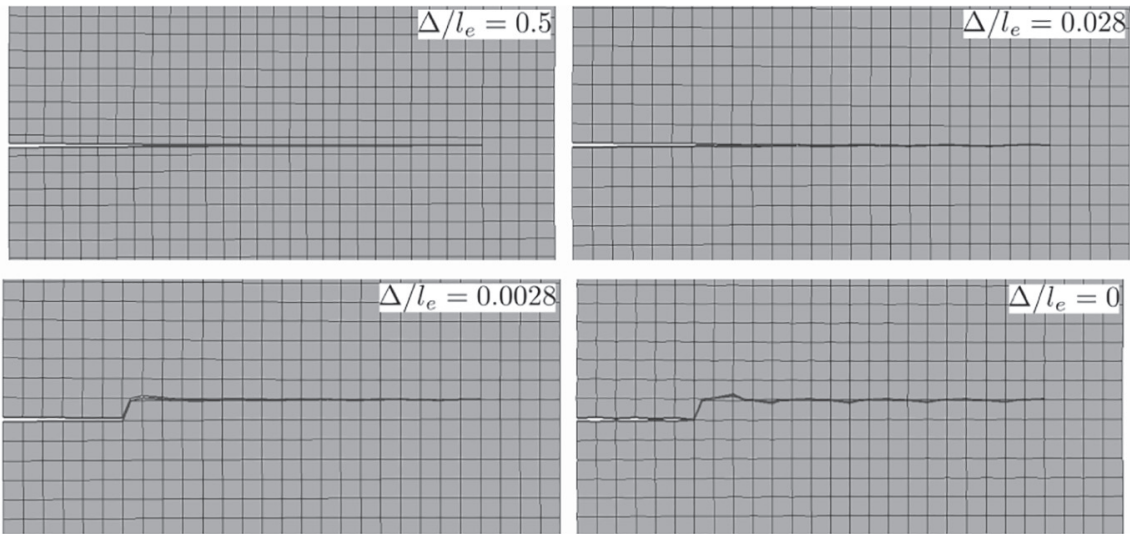

(a) Peak force state.
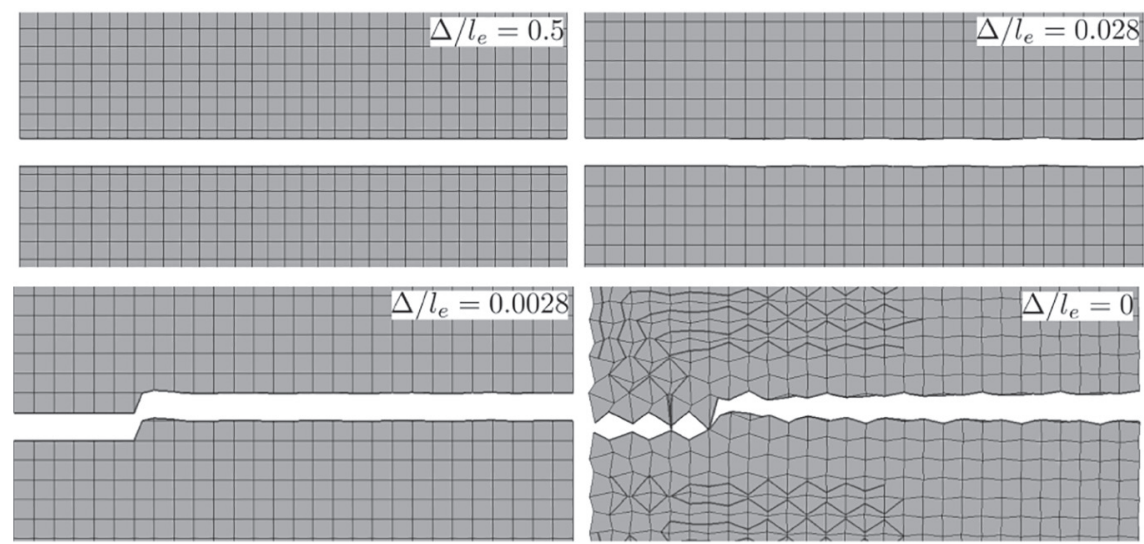

(b) Final state.

Fig. 25. Crack pattern of the solid plate containing an initial precrack of length $a=2 \mathrm{~mm}$ (deformation scale factor: $\times 20$ ). (a) Peak force and (b) Final state.
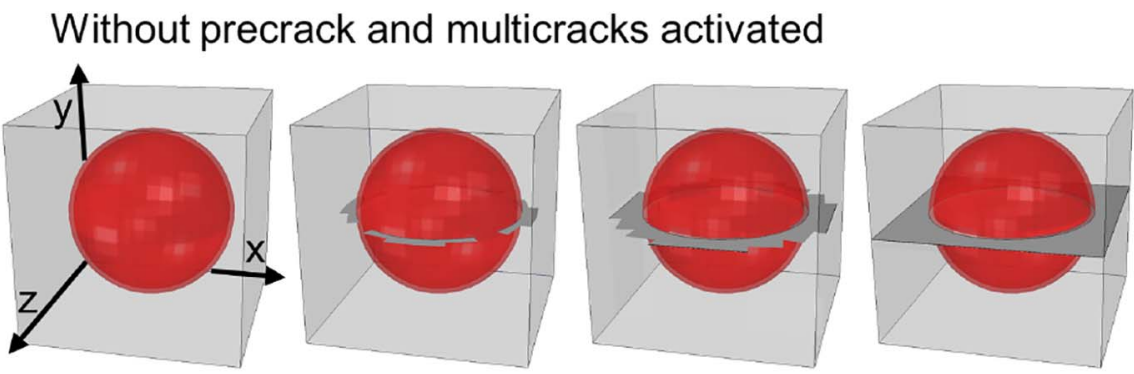

With precrack and multicracks activated
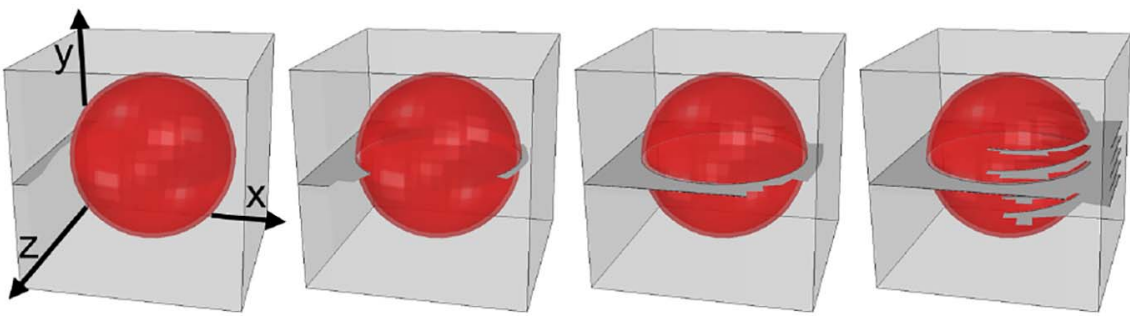

Fig. 26. Crack evolution without and with precrack when multicracks is activated. 
future, the beam is decomposed in two regions: (i) a central region that contains the embedded glass capsules, where XFEM and cohesive surfaces are used and (ii) two lateral regions, or wings, described by conventional FEM. Both regions are conveniently tied to fulfill the material continuity of the beam. A thorough convergence analysis of the mesh has been performed in order to ensure the reliability of the results. Two mechanical aspects are studied: the role of the insertion of capsules in the reinforcement or weakening effect of the overall beam strength under bending, and the analysis of which interface strength and capsule size are more beneficial to make the capsules break properly (and deliver the healing agent into the crack). Therefore, this model is restricted to analyze the breakage of the capsules, where the capsules are assumed empty. Given a fixed external capsule radius of $1.5 \mathrm{~mm}$ (commonly used in lab tests), the model predicts that using capsules with $t / R<0.12$, a minimum interface strength of $2 \mathrm{MPa}$ has to be ensured to break and liberate the healing agent. If this ratio increases, the required minimum bonding strength also increases, but in a non-linear way. If this increase of interface strength cannot be technically achieved in the lab (e.g., by capsule surface treatment), the self-healing strategy will not take effect, as the capsule will debond from the matrix. However, a non-negligible strengthening of the beam can be obtained as side-effect due to the reinforcement introduced by the capsules. On the other hand, the effect of the number of capsules and the clustering have also been analyzed. A number of capsules between 1 and 8 has been progressively located close to the notch of the beam. As a result, a light increase of the peak force in bending is observed (compared to the bending response of a solid beam). Conversely, it has been noted the following trend: in regions where the capsules are densely clustered, the concrete material is more susceptible to fail sooner despite the reinforcement effect provided by the capsules. Therefore, this result might indicate that the global beam response might suffer an important weakening if the capsular interdistance decreases sufficiently.

Regarding the material system that involves polymers, an encapsulation-based self-healing polymer has been studied using a simple micro-scale model that makes use of a Representative Volume Element (RVE). Similarly to the previous macro-scale model, the damage has been introduced with the combination of XFEM elements in both materials, the polymer matrix and the shell capsule. Both constituents are joined by using cohesive surfaces. The results show that both types of damages, the material fracture and the capsule debonding, can coexist for the studied range of mechanical properties. The limit of breakage and debonding of micro-capsules has been studied without and with a preexisting crack. In terms of global mechanical response, it is found that both scenarios provide indistinguishable homogenized stress-strain curves, despite the fact that both crack sequence and crack pattern are different. The overall material properties of the self-healing polymer have been calculated for a wide range of volume fractions and different capsule geometric ratios. Despite the quantitative differences between the experimental available data and the micro-model predictions (due to simplifications), it has been found a very good agreement from the qualitative point of view. As a matter of fact, the present model catches very well the trend of variation of the elasticity and stress at failure as a function of the volume fraction.

To finish, a note of caution. An important advantage provided by the present commercial implementation is its capability of predicting arbitrary crack paths in any specimen under load. This capability is undoubtedly a huge help for researchers to understand and to predict the global response obtained from real experiments. That being said, it is worth mentioning to know and bear in mind the limitations explained in this work, because they might represent an important bias. These biases can introduce some uncontrolled or hardly detectable artifacts in the final results that hinder their interpretation for further applications. This situation might become even worse when the model is particularly elaborated and its physical response is far from being intuitive or predictable.

\section{Acknowledgments}

Financial support from the Strategic Initiative Materials - Flanders (program Engineered Self-Healing materials (SHE) - projects SECEMIN and SEPOCOM and program H-INT-S) is gratefully acknowledged.

\section{References}

[1] S.R. White, N.R. Sottos, P.H. Geubelle, J.S. Moore, M.R. Kessler, S.R. Sriram, E.N. Brown, S. Viswanathan, Autonomic healing of polymer composites, Nature 409 (6822) (2001) 794-797.

[2] S. Van der Zwaag, N. Van Dijk, H. Jonkers, S. Mookhoek, W. Sloof, Self-healing behaviour in man-made engineering materials: bioinspired but taking into account their intrinsic character, Philosophical Transactions of the Royal Society of London A: Mathematical, Physical and Engineering Sciences 367 (1894) (2009) 1689-1704.

[3] K. Van Tittelboom, N. De Belie, D. Van Loo, P. Jacobs, Self-healing efficiency of cementitious materials containing tubular capsules filled with healing agent, Cem. Concr. Compos. 33 (4) (2011) 497-505.

[4] M. Wu, B. Johannesson, M. Geiker, A review: self-healing in cementitious materials and engineered cementitious composite as a Self-Healing Material, Constr. Build. Mater. 28 (1) (2012) 571-583.

[5] K. Van Tittelboom, N. De Belie, Self-healing in cementitious materials - a review, Materials 6 (6) (2013) 2182-2217.

[6] M. Kessler, N. Sottos, S. White, Self-healing structural composite materials, Compos. A: Appl. Sci. Manuf. 34 (8) (2003) 743-753.

[7] J. Tirosh, A.S. Tetelman, Fracture conditions of a crack approaching a disturbance, Int. J. Fract. 12 (2) (1976) 187-199.

[8] M.G. Knight, L.C. Wrobel, J.L. Henshall, L.A. De Lacerda, A study of the interaction between a propagating crack and an uncoated/coated elastic inclusion using the be technique, Int. J. Fract. 114 (1) (2002) 47-61.

[9] R. Kitey, H.V. Tippur, Dynamic crack growth past a stiff inclusion: optical investigation of inclusion eccentricity and inclusion-matrix adhesion strength, Exp. Mech. 48 (1) (2008) 37-53.

[10] K.C. Jajam, H.V. Tippur, Role of inclusion stiffness and interfacial strength on dynamic matrix crack growth: an experimental study, Int. J. Solids Struct. 49 (9) (2012) 1127-1146.

[11] A.C. Balazs, Modeling self-healing materials, Mater. Today 10 (9) (2007) 18-23.

[12] S.V. Zemskov, H.M. Jonkers, F.J. Vermolen, Two analytical models for the probability characteristics of a crack hitting encapsulated particles: application to self-healing materials, Comput. Mater. Sci. 50 (12) (2011) 3323-3333.

[13] H. Huang, G. Ye, The effects of capules on self-healing efficiency in cementitious materials, Second International Conference on Microstructural-Related Durability of Cementitious Composites, Rilem Proceedings Pro083, 11-13 April, 2012, Amsterdam, The Netherlands, Rilem, 2012, pp. 1090-1100.

[14] Z. Lv, H. Chen, Analytical models for determining the dosage of capsules embedded in self-healing materials, Comput. Mater. Sci. 68 (2013) 81-89.

[15] F.A. Gilabert, D. Garoz, W. Van Paepegem, Stress concentrations and bonding strength in encapsulation-based self-healing materials, Mater. Des. 67 (2015) 28-41.

[16] G.B. Pye, J.J. Beaudoin, An energy approach to bond strength determinations in cement systems, Cem. Concr. Res. 22 (4) (1992) 551-558.

[17] J. Zhou, M. Huang, F. Sagnang, W. Soboyejo, Interfacial failure of a dental cement composite bonded to glass substrates, Dent. Mater. 22 (6) (2006) 585-591.

[18] J. Bieniaś, H. Dębski, B. Surowska, T. Sadowski, Analysis of microstructure damage in carbon/epoxy composites using FEM, Comput. Mater. Sci. 64 (2012) 168-172.

[19] L. Bouhala, A. Makradi, S. Belouettar, H. Kiefer-Kamal, P. Fréres, Modelling of failure in long fibres reinforced composites by X-FEM and cohesive zone model, Compos. Part B 55 (2013) 352-361.

[20] H. Wang, J. Wang, J. Chen, Micromechanical analysis of asphalt mixture fracture with adhesive and cohesive failure, Eng. Fract. Mech. 132 (2014) 104-119.

[21] D.M. Grogan, C.M.Ó. Brádaigh, S.B. Leen, A combined XFEM and cohesive zone model for composite laminate microcracking and permeability, Compos. Struct. 120 (2015) 246-261.

[22] Abaqus, 2016 User documentation, Tech. Rep. Dassault Systémes Simulia Corp., Providence, RI, USA. 2016.

[23] J.H. Song, P.M.A. Areias, T. Belytschko, A method for dynamic crack and shear band propagation with phantom nodes, Int. J. Numer. Methods Eng. 67 (6) (2006) 868-893.

[24] J.J.C. Remmers, R. de Borst, A. Needleman, The simulation of dynamic crack propagation using the cohesive segments method, J. Mech. Phys. Solids 56 (1) (2008) 70-92.

[25] S. Osher, J.A. Sethian, Fronts propagating with curvature-dependent speed: algorithms based on Hamilton-Jacobi formulations, J. Comput. Phys. 79 (1) (1988) $12-49$.

[26] M. Stolarska, D.L. Chopp, N. Moës, T. Belytschko, Modelling crack growth by level sets in the extended finite element method, Int. J. Numer. Methods Eng. 51 (8) (2001) 943-960.

[27] N. Moës, A. Gravouil, T. Belytschko, Non-planar 3D crack growth by the extended finite element and level sets-Part I: mechanical model, Int. J. Numer. Methods Eng. 53 (11) (2002) 2549-2568.

[28] M. Duflot, A study of the representation of cracks with level sets, Int. J. Numer. Methods Eng. 70 (11) (2007) 1261-1302.

[29] G.T. Camacho, M. Ortiz, Computational modelling of impact damage in brittle 
materials, Int. J. Solids Struct. 33 (20-22) (1996) 2899-2938.

[30] P.H. Geubelle, J.S. Baylor, Impact-induced delamination of composites: a 2D simulation, Compos. Part B 29 (5) (1998) 589-602.

[31] A. Turon, C. Dávila, P. Camanho, J. Costa, An engineering solution for mesh size effects in the simulation of delamination using cohesive zone models, Eng. Fract. Mech. 74 (10) (2007) 1665-1682.

[32] K. Park, G.H. Paulino, Cohesive zone models: a critical review of tractionseparation relationships across fracture surfaces, Appl. Mech. Rev. 64 (6) (2013) 060802.

[33] M.L. Benzeggagh, M. Kenane, Measurement of mixed-mode delamination fracture toughness of unidirectional glass/epoxy composites with mixed-mode bending apparatus, Compos. Sci. Technol. 56 (4) (1996) 439-449.

[34] Q.V. Bui, A Modified Benzeggagh-Kenane fracture criterion for mixed-mode delamination, J. Compos. Mater. (2010) 389-413.

[35] A. Turon, P. Camanho, J. Costa, C. Dávila, A damage model for the simulation of delamination in advanced composites under variable-mode loading, Mech. Mater. 38 (11) (2006) 1072-1089.

[36] K. Van Tittelboom, K. Adesanya, P. Dubruel, P. Van Puyvelde, N. De Belie, Methyl methacrylate as a healing agent for self-healing cementitious materials, Smart Mater. Struct. 20 (122) (2011) 125016/1-12.

[37] W.Y. Sun L., Q.G. Yu, Experimental research on the self-healing performance of micro-cracks in concrete bridge, Adv. Mater. Res. 250-253 (2011) 28-32.

[38] M. Maes, K. Van Tittelboom, N. De Belie, The efficiency of self-healing cementitious materials by means of encapsulated polyurethane in chloride containing environments, Constr. Build. Mater. 71 (2014) 528-537.

[39] E. Tsangouri, G. Karaiskos, D.G. Aggelis, A. Deraemaeker, D. Van Hemelrijck, Crack sealing and damage recovery monitoring of a concrete healing system using embedded piezoelectric transducers, Struct. Health Monit. (2015).

[40] M.L. Gambhir, Concrete Technology. Civil engineering series, Tata McGraw-Hill Pub., 9780070583740, 2004.

[41] J. Mencik, Strength and Fracture of Glass and Ceramics, Elsevier Science and Technology, Oxford, United Kingdom, 1992.

[42] J. Shackelford, R. Doremus, Ceramic and Glass Materials: Structure, Properties and Processing, Springer, 9781441944603, 2010.

[43] Y.S. Jenq, S.P. Shah, A fracture toughness criterion for concrete, Eng. Fract. Mech. 21 (5) (1985) 1055-1069.

[44] B.L. Karihaloo, P. Nallathambi, Effective crack model for the determination of fracture toughness (KIce) of concrete, Eng. Fract. Mech. 35 (4) (1990) 637-645.

[45] S.A. Hamoush, H. Abdel-Fattah, The fracture toughness of concrete, Eng. Fract. Mech. 53 (3) (1996) 425-432.

[46] M. Overend, G. Parke, D. Buhagiar, Predicting failure in glass - a general crack growth model, J. Struct. Eng. 133 (8) (2007) 1146-1155.

[47] F.A. Veer, P.C. Louter, F.P. Bos, The strength of annealed, heat-strengthened and fully tempered float glass, Fatigue Fract. Eng. Mater. Struct. 32 (1) (2009) 18-25.

[48] J. Belis, Kipsterkte Van Monolithische En Gelamineerde Glazen Liggers, Ph.D. thesis Ghent University, 2005, http://dx.doi.org/1854/5844.

[49] K.R. Linger, D.G. Holloway, The fracture energy of glass, Philos. Mag. 18 (156) (1968) 1269-1280.

[50] S.M. Wiederhorn, Fracture surface energy of glass, J. Am. Ceram. Soc. 52 (2) (1969) 99-105.

[51] K. Van Tittelboom, Self-Healing Concrete Through Incorporation of Encapsulated Bacteria- or Polymer-Based Healing Agents, Ph.D. thesis Ghent University, 2012.

[52] F.A. Gilabert, K. Van Tittelboom, E. Tsangouri, D. Van Hemelrijck, N. De Belie, W. Van Paepegem, Determination of strength and debonding energy of a glassconcrete interface for encapsulation-based self-healing concrete, Cem. Concr. Compos. 79 (2017) 76-93.

[53] A. Hillerborg, M. Modéer, P.E. Petersson, Analysis of crack formation and crack growth in concrete by means of fracture mechanics and finite elements, Cem. Concr. Compos. 6 (6) (1976) 773-781.

[54] N. Moës, T. Belytschko, Extended finite element method for cohesive crack growth, Eng. Fract. Mech. 69 (7) (2002) 813-833.

[55] P. Grassl, M. Jirásek, Damage-plastic model for concrete failure, Int. J. Solids
Struct. 43 (22-23) (2006) 7166-7196.

[56] K. Song, C.G. Dávila, C.A. Rose, Guidelines and parameter selection for the simulation of progressive delamination, Conference Proceedings 2008 Abaqus Users' Conference: May 19-22, Newport, Rhode Island, USA, Dassault Systemes, 2008, 2008, pp. 1-15.

[57] E. Tsangouri, D.G. Aggelis, K. Van Tittelboom, N. De Belie, D. Van Hemelrijck, Detecting the activation of a self-healing mechanism in concrete by acoustic emission and digital image correlation, Sci. World J. 2013 (2013) Article ID 424560(1-20).

[58] J. Feiteira, E. Tsangouri, E. Gruyaert, C. Lors, G. Louis, N. De Belie, Monitoring crack movement in polymer-based self-healing concrete through digital image correlation, acoustic emission analysis and sem in-situ Loading, Mater. Des. (2016).

[59] S.A. Ponnusami, S. Turteltaub, S. van der Zwaag, Cohesive-zone modelling of crack nucleation and propagation in particulate composites, Eng. Fract. Mech. 149 (2015) 170-190.

[60] Y.C. Yuan, T. Yin, M.Z. Rong, M.Q. Zhang, Self healing in polymers and polymer composites. Concepts, realization and outlook: a review, eXPRESS Polymer Letters 2 (4) (2008) 238-250.

[61] S.R. White, N.R. Sottos, P.H. Geubelle, J.S. Moore, M.R. Kessler, S.R. Sriram, E.N. Brown, S. Viswanathan, Autonomic healing of polymer composites, Nature 409 (6822) (2001) 794-797.

[62] M. Yu, P. Zhu, Y. Ma, Effects of particle clustering on the tensile properties and failure mechanisms of hollow spheres filled syntactic foams: a numerical investigation by microstructure based modeling, Mater. Des. 47 (2013) 80-89.

[63] G.G. Sozhamannan, S.B. Prabu, R. Paskaramoorthy, Failures analysis of particle reinforced metal matrix composites by microstructure based models, Mater. Des. 31 (8) (2010) 3785-3790.

[64] S.D. Mookhoek, H.R. Fischer, S. v. d. Zwaag, A numerical study into the effects of elongated capsules on the healing efficiency of liquid-based systems, Comput. Mater. Sci. 47 (2) (2009) 506-511.

[65] E.N. Brown, S.R. White, N.R. Sottos, Microcapsule induced toughening in a selfhealing polymer composite, J. Mater. Sci. 39 (5) (2004) 1703-1710.

[66] X.K.D. Hillewaere, R.F.A. Teixeira, L.T.T. Nguyen, J.A. Ramos, H. Rahier, F.E.D.u. Prez, Autonomous self-h of epoxy thermosets with thiol-isocyanate chemistry, Adv. Funct. Mater. 24 (35) (2014) 5575-5583.

[67] Y.C. Yuan, M.Z. Rong, M.Q. Zhang, J. Chen, G.C. Yang, X.M. Li, Self-healing polymeric materials using epoxy/mercaptan as the healant, Macromolecules 41 (14) (2008) 5197-5202.

[68] D. Garoz, F.A. Gilabert, R.D.B. Sevenois, S.W.F. Spronk, A. Rezaei, W. Van Paepegem, Definition of periodic boundary conditions in explicit dynamic simulations of micro- or meso-scale unit cells with conformal and non-conformal meshes, 17th European Conference on Composite Materials, European Society for Composite Materials (ESCM), 2016, pp. 1-6.

[69] M.W. Keller, N.R. Sottos, Mechanical properties of microcapsules used in a selfhealing polymer, Exp. Mech. 46 (6) (2006) 725-733.

[70] Matweb, Overview of Materials for Epoxy and Urea-Formaldehyde, (2016) http:// www.matweb.com.

[71] R.O. Ebewele, B.H. River, G.E. Myers, Polyamine-modified urea-formaldehydebonded wood joints. III. Fracture toughness and cyclic stress and hydrolysis resistance, J. Appl. Polym. Sci. 49 (2) (1993) 229-245.

[72] T. Hobbiebrunken, B. Fiedler, M. Hojo, M. Tanaka, Experimental determination of the true epoxy resin strength using micro-scaled specimens, Compos. A: Appl. Sci. Manuf. 38 (3) (2007) 814-818.

[73] E.N. Brown, M.R. Kessler, N.R. Sottos, S.R. White, In situ poly(ureaformaldehyde) microencapsulation of dicyclopentadiene, J. Microencapsul. 20 (6) (2003) 719-730.

[74] E.N. Brown, S.R. White, N.R. Sottos, Retardation and repair of fatigue cracks in a microcapsule toughened epoxy composite-part II: in situ self-healing, Compos. Sci. Technol. 65 (15-16) (2005) 2474-2480.

[75] F.A. Gilabert, D. Garoz, W. Van Paepegem, Numerical study of transitional brittleto-ductile debonding of a capsule embedded in a matrix, Compos. Interfaces 24 (1) (2017) 69-84. 